\title{
2 Effect of Brightness of Visual Stimuli on EEG Signals
}

\author{
3 Kübra Eroğlu ${ }^{1 *}$, Temel Kayıkçığlu ${ }^{2}$ and Onur Osman ${ }^{1}$ \\ 41 Istanbul Arel University 1; kubraeroglu@arel.edu.tr, onurosman@arel.edu.tr, Istanbul 34537, Turkey \\ 52 Karadeniz Technical University 2; tkayikci@ktu.edu.tr, Trabzon 61080, Turkey \\ 6 * Correspondence: kubraeroglu@arel.edu.tr; Tel.: +90 2128672500 - 1425 \\ $7 \quad \uparrow$ These authors contributed equally to this work
}

Abstract: The aim of this study was to examine brightness effect, which is the perceptual property of visual stimuli, on brain responses obtained during visual processing of these stimuli. For this purpose, brain responses of the brain to changes in brightness were explored comparatively using different emotional images (pleasant, unpleasant and neutral) with different luminance levels. Moreover, electroencephalography recordings from 12 different electrode sites of 31 healthy participants were used. The power spectra obtained from the analysis of the recordings using short time Fourier transform were analyzed, and a statistical analysis was performed on features extracted from these power spectra. Statistical findings obtained from electrophysiological data were compared with those obtained from behavioral data. The results showed that the brightness of visual stimuli affected the power of brain responses depending on frequency, time and location. According to the statistically verified findings, the distinctive effect of brightness occurred in the parietal and occipital regions for all the three types of stimuli. Accordingly, the increase in the brightness of pleasant and neutral images increased the average power of responses in the parietal and occipital regions whereas the increase in the brightness of unpleasant images decreased the average power of responses in these regions. However, the increase in brightness for all the three types of stimuli reduced the average power of frontal and central region responses (except for 100$300 \mathrm{~ms}$ time window for unpleasant stimuli). The statistical results obtained for unpleasant images were found to be in accordance with the behavioral data. The results also revealed that the brightness of visual stimuli could be represented by changing the activity power of the brain cortex. The main contribution of this research was to comprehensively examine brightness effect on brain activity for images with different emotional content and different frequency bands at different time windows of visual processing for different brain regions. The findings emphasized that the brightness of visual stimuli should be viewed as an important parameter in studies using emotional image techniques such as image classification, emotion evaluation and neuro-marketing.

Keywords: EEG; luminance; brightness; IAPS; STFT; feature extraction; visual processing; emotion.

\section{Introduction}

Visual processing is a complex network that requires the effectiveness of multiple frequencies at different time windows and the parallel activity of different brain regions. In this complex network many electrical activities occurs in our brain. Many researchers have shown great interest to investigate the nature of these activities, how they are formed and by which parameters (emotional content, color, spatial frequency, saturation, hue and brightness, etc.) they are affected. In many studies on this subject, the effect of the emotional content of visual scenes was investigated on brain activity during visual processing [1-9]. In these studies, images in the International Emotional Image System database [10] with different emotional contents (pleasant, unpleasant and neutral) were widely used. The International Affective Picture System (IAPS) database contains standardized emotional images for use in experiments. These images are standardized according to the parameters 
of arousal, emotional valence and dominance. For this reason, they are often used in experimental psychology research, especially for investigation of emotional processes.

Many studies investigating the effects of the emotional content of IAPS scenes on brain activity have focused on event related potential (ERP) responses observed in electroencephalography (EEG). The common finding in most of these studies is that the brain responds differently to unpleasant or pleasant stimuli than to neutral stimuli [11-16]. However, in some IAPS studies, different effects of valence and arousal on electrical activity have been reported [1, 17-20]. It has been noted that valence modulates ERP components in an early time period (before $200 \mathrm{~ms}$ ) whereas arousal often modulates in a later time period (after $250 \mathrm{~ms}$ ) [20-22]. In addition, in many studies, different effects of IAPS images in different frequency bands have been mentioned [11, 23-25].

When these and similar studies in the literature are examined, it is realized that the emotional content of visual stimuli modulates brain activity during visual processing. This effect has been observed in various frequency bands and brain regions in the early and late time period. However, the luminance values of the images in the IAPS database differ from one to another and it is not known how this difference in luminance affects brain responses during visual processing. We consider that brightness, which is suggested to be one of the most important sources of perceptual processing [26] may have a strong effect on brain activity during visual processing. Apart from the content of visual stimuli, the brightness of visual stimuli may also have the potential to influence neural activity.

In many studies, brightness effect was evaluated on visual processing with different types of stimuli and different perspectives [27-34]. For instance, in a study, in which unilaterally flashed bar stimuli were used, the stimulus luminance was reported to have a higher ERP amplitude in the regions of the posterior $\mathrm{N} 95$ (80-110 ms), the occipital Pl (110-140 ms) and the parietal Nl (130-180 ms) [28]. In a study without EEG and with use of the pleasure-arousal-dominance emotion model, saturation and brightness were declared to have strong and consistent effects on emotions [30]. In another study without EEG, behavioral analyses were performed, the brightness levels of IAPS images were found to be correlated with the valence degrees of the images. In the study, it was specified that dark IAPS images were evaluated more negatively, while bright IAPS images were evaluated more positively [35]. These and similar studies have demonstrated clear evidence that the brightness of stimuli has an impact on both behavioral data and as well as visual and emotional processes. Therefore, it is important to investigate whether brightness modulates brain oscillations in response to emotional visual stimuli and how this occurs.

In a few recent studies, the luminance values of visual stimuli have been kept constant at a certain value to avoid possible brightness [36-43]. However, it is not clear whether the brightness of visual stimuli influences brain activity in response to these stimuli and if whether there is any interaction between them. Possible brightness may affect our brain activity during visual processing and may direct our perceptions or cognitive processes.

A group of authors in a study investigated the brightness effect of neutral and erotic IAPS images on only two components (N2 and EPN). In their study, they reported that original erotic scenes exhibited greater N1 amplitude than bright scenes [36]. In our specific study on the subject, we investigated brightness effect only for the delta frequency band. We found that bright unpleasant images elicited lower delta response compared to original unpleasant images [44]. However, the effect of the brightness of stimuli on visual processing needs to be examined in more detail and multidimensionally. Specific stimulus types have been used in the relevant brain studies, and the brightness effect has been investigated for specific ERP components and specific frequency bands. In the literature, there is no integrative study investigating the effect of brightness of emotional images with different content on electrophysiological responses in terms of time, frequency and location.

A possible interaction between the brightness of visual stimuli and neural activity can lead to misinterpretation of sensory and cognitive processes (perception, decision making, selective attention, communication with memory, etc.), depending on the type of effect and the time and frequency range at which it occurs. This situation reveals the necessity of controlling the brightness 
of the stimuli in studies using emotion classification, emotion evaluation, neuro-marketing and braincomputer interface.

The aim of this study was to investigate the brightness effect of pleasant, unpleasant and neutral IAPS images on electrophysiological responses during visual processing. For this purpose, power spectrograms of electrophysiological responses obtained upon the presentation of original and bright versions of pleasant, unpleasant and neutral IAPS images were obtained using short time Fourier transform (STFT). Then, the features extracted from these power spectrograms were compared statistically between the original and bright groups for three different emotional contents. After comparison, frequency bands, time windows and locations where brightness effect was observed were determined. In addition to all analyses, the behavioral data obtained for all the IAPS images (both the original and bright versions) from each individual participating in the study were statistically evaluated. Thus, brightness effect was investigated in parallel on both electrophysiological and behavioral responses.

Our hypotheses were as follows;

1- During visual processing, electrophysiological responses can be affected by the brightness of stimuli;

2- The brightness of different emotional stimuli (pleasant, unpleasant, and neutral) cause different effects on brain activity;

3- All these effects vary according to time window, frequency band and brain region, in which electrophysiological responses occur, and these changes can be examined by power spectrograms of electrophysiological responses.

By evaluation of these hypotheses, the effect of the brightness of stimuli will be determined on visual processing with respect to time, frequency and location. In this way, we can provide researchers with information about parameters to be considered in studies using visual stimuli (visual processing, emotional image classification, emotion evaluation, brain-computer interfaces, neuro-marketing, etc.). The results of the study will add to the previous literature on brain dynamics underlying visual processing and parameters that affect these dynamics.

\section{Materials and Methods}

\subsection{Participants}

Thirty-one healthy participants (17 females and 14 males) participated in the study. The average age of the participants was $27.33 \pm 7.03$. The participants were volunteers who did not have any psychiatric or neurological disorders or skin diseases, did not use medications that affect the nervous system, and had a score of 17 or lower on the Beck depression scale, which is an inventory consisting of 21 items on the emotional, cognitive, and motivational states of depression.

All participants gave their informed consent for inclusion before they participated in the study. This study was conducted after the approval of the Ethics Committee of Istanbul Arel University by decision no 10432314-200.00.00-18.

\subsection{Stimuli}

Thirty color images, consisting of 10 unpleasant images (wild animals, cut limbs, etc.), 10 pleasant images (babies, cute animals, etc.) and 10 neutral images (human faces, household goods, etc.), were used in the study. All the images were obtained from the IAPS database. This database is frequently used in emotion processing studies using visual stimuli. By changing the brightness level of each image, two different image groups with low and high luminance values were obtained (original images and bright images). For this purpose, firstly, the average power of each image was calculated and multiplied by an appropriate positive constant (constant $>1$ ) to increase the brightness of the image. During this process, the intensity values were normalized before the images were saved as jpeg files to avoid saturation. Afterwards, the band limited contrast value (C) was calculated for each image and kept constant $[45,46]$. 
148

$$
C(u, v)=2 A(u, v) / d c
$$

149

150

151

152

153

154

155

$\mathrm{A}(\mathrm{u}, \mathrm{v})$ is the Fourier transform amplitude of the image, $\mathrm{u}$ and $\mathrm{v}$ are the horizontal-vertical spatial frequency coordinates and $\mathrm{dc}$ is the zero frequency component. The luminance values of both sets of images were measured with the 'Delta OHM HD 2302.0 Luminance Meter'. The code numbers and luminance values of the original and bright image groups are shown in Table 1.

Table 1. The code numbers, luminance values and increased luminance values of the pleasant, unpleasant and neutral images.

\begin{tabular}{|c|c|c|c|}
\hline$\frac{\text { Emotional }}{\underline{\text { Content }}}$ & $\begin{array}{l}\text { Image } \\
\text { Code } \\
\text { Number }\end{array}$ & $\begin{array}{c}\text { Luminance Values of } \\
\text { Images (original- } \\
\text { images) } \mathrm{cd} / \mathrm{m}^{2}\end{array}$ & $\begin{array}{l}\text { Increased Luminance } \\
\text { Values of Images } \\
\text { (bright-images) } \mathrm{cd} / \mathrm{m}^{2}\end{array}$ \\
\hline \multirow[t]{10}{*}{ Pleasant } & 1460 & 24.2 & 50.4 \\
\hline & 1500 & 16.9 & 34.4 \\
\hline & 1710 & 45.0 & 93.5 \\
\hline & 1722 & 26.7 & 55.7 \\
\hline & 1750 & 30.3 & 61.6 \\
\hline & 2058 & 49.5 & 99.4 \\
\hline & 2080 & 43.5 & 88.9 \\
\hline & 2165 & 40.6 & 84.4 \\
\hline & 2332 & 22.9 & 47.0 \\
\hline & 2340 & 25.0 & 51.3 \\
\hline \multicolumn{2}{|c|}{$($ mean \pm SD $)$} & $32.5 \pm 10.6$ & $66.7 \pm 21.6$ \\
\hline \multirow[t]{10}{*}{ Unpleasant } & 1052 & 36.2 & 74.1 \\
\hline & 1114 & 25.8 & 52.0 \\
\hline & 1274 & 25.5 & 52.8 \\
\hline & 1930 & 20.5 & 41.2 \\
\hline & 1932 & 24.6 & 50.3 \\
\hline & 2205 & 43.8 & 88.5 \\
\hline & 2352 & 54.2 & 111.2 \\
\hline & 3400 & 30.3 & 61.2 \\
\hline & 6350 & 38.5 & 77.6 \\
\hline & 6550 & 25.1 & 51.8 \\
\hline \multicolumn{2}{|c|}{$($ mean \pm SD $)$} & $32.5 \pm 10.0$ & $66.1 \pm 20.5$ \\
\hline \multirow[t]{10}{*}{ Neutral } & 2190 & 17.6 & 35.3 \\
\hline & 2210 & 30.7 & 64.4 \\
\hline & 2214 & 55.7 & 115.9 \\
\hline & 2480 & 17.2 & 34.4 \\
\hline & 2495 & 26.0 & 54.4 \\
\hline & 7010 & 25.6 & 51.2 \\
\hline & 7020 & 53.8 & 111.7 \\
\hline & 7030 & 37.4 & 76.6 \\
\hline & 7041 & 28.5 & 59.1 \\
\hline & 7080 & 31.5 & 73.5 \\
\hline \multicolumn{2}{|c|}{$($ mean \pm SD $)$} & $32.4 \pm 12.6$ & $67.7 \pm 26.6$ \\
\hline
\end{tabular}


The one-way ANOVA test was used to compare the luminance level of the original and bright versions of emotional images. According to the one-way ANOVA test results, there was no difference between the luminance values of the pleasant, unpleasant and neutral images with the same brightness level $(\mathrm{F}(2,29)=0.00, \mathrm{p}=1.00$ and $\mathrm{F}(2,29)=0.01, \mathrm{p}=0.98$, respectively). The mean and SD values of all the stimuli are shown in Table 1.

\subsection{Experimental Design}

In this study, an experiment was designed for all the participants using two image groups (original and bright image groups), which included pleasant, unpleasant and neutral image. For the experiment, all the participants were seated 1 meter $(\mathrm{m})$ away from a 19-inch computer screen with a refresh rate of $60 \mathrm{~Hz}$ in a dark room. The distance between the screen and the eyes of the subjects was set to $1 \mathrm{~m}$, since the luminance was measured by placing the light meter $1 \mathrm{~m}$ away from the computer screen. IAPS images were displayed on the screen in a random sequence. The block design (10 unpleasant images x 4 loops, 10 pleasant images x 4 loops, 10 neutral images x 4 loops) was used during this process [43]. In each experiment, order of the presentation of stimuli was counterbalanced across the subjects. All the images were displayed for $1000 \mathrm{~ms}$ with the stimulus interval of 3-7 s. This process was carried out for both the bright and original images. During the process, EEG recordings were taken from all the participants.

\subsection{Electroencephalography (EEG) Recordings}

The EEG recordings were obtained in the Psychophysiology Laboratory of Istanbul Arel University. The laboratory environment was isolated using Faraday cages. In the study, the EEG recordings were obtained from 12 different electrode sites $\left(F_{3}, F_{z}, F_{4}, C_{3}, C_{z}, C_{4}, P_{3}, P_{z}, P_{4}, O_{1}, O_{z}\right.$ and $\mathrm{O}_{2}$ ) from the subjects. The electrodes were placed on an EASYCAP according to the international 1020 system. Earlobe electrodes $\left(\mathrm{A}_{1}\right.$ and $\left.\mathrm{A}_{2}\right)$ were used as a reference. In addition, electrooculogram (EOG) signals were recorded from the right eye, medial upper and lateral orbital rim. $\mathrm{Ag} / \mathrm{AgCl}$ electrodes were used for the EOG recordings and reference electrodes. All electrode impedances were selected to be less than $10 \mathrm{k} \Omega$. The EEG recordings were enhanced by a BrainAmp 32-channel DC amplifier with a band limit of 0.01-250 Hz. The sampling frequency was $500 \mathrm{~Hz}$. In the pleasant, unpleasant and neutral stimulus conditions, sweep numbers were randomly equalized. By reviewing the EOG recordings, epochs with eye movements or blink artifacts were cleaned offline.

\subsection{Behavioral Data}

The IAPS contains the subjective rating of emotional valence and arousal with the use of a rating scale, the Self-Assessment-Manikin [10]. At the end of the experiment, the scale was applied to each subject to test the subjective rating of the emotional valence (pleasant/ unpleasant/ neutral) and arousal (aroused/ relaxed) value of the images in the original and bright groups [47][48]. By showing each image again to each person, all the images were evaluated for valence (pleasant/ unpleasant/ neutral) and arousal (aroused/ relaxed) dimensions with a nine-point scale. The brightness rating was also assessed by asking which of the two images was brighter. All the subjects answered all the evaluation questions correctly.

\subsection{Pre-Processing of Electroencephalography (EEG) Data}

The EEG recordings were divided into sub-data sets according to the content of the images in the bright and original groups used during the experiment (ORIGINALpleasant, ORIGINALunpleasant, ORIGINALneutral, BRIGHT pleasant, BRIGHT unpleasant and BRIGHT neutral). Each sub-data set contained EEG recordings from 12 channels of 31 subjects and consisted of $1500 \mathrm{~ms}$ epochs, which were $500 \mathrm{~ms}$ before the visual stimulus onset and $1000 \mathrm{~ms}$ after the visual stimulus onset. Fifth-order notch and median filters were used to clean the $50 \mathrm{~Hz}$ network noise and other noises. The EEG recordings were highly noisy, in which many amplitude differences were observed between the subjects. With normalization, data from different scales can be converted to the same scale. Thus, all data become 
comparable on the same scale. In this study, the Min-Max normalization method [47] was used in (2). All the EEG epochs were scaled in the range of $[-111]$ with this procedure.

$$
Y_{n}=2 *\left(\frac{Y-Y_{\min }}{Y_{\max }-Y_{\min }}\right)-1
$$

where $Y$ denotes the original EEG epoch, $Y_{\min }$ is the minimum value in $Y, Y_{\max }$ is the maximum value in $Y$ and $Y_{n}$ is the normalized epoch.

\subsection{Electroencephalography (EEG) Data Analysis}

Time-amplitude and time-frequency analyses of the EEG recordings were performed in each sub-data set. In these evaluations, time windows, in which brightness effect was observed, were determined and features were extracted for the time windows.

\subsubsection{Time-Amplitude Analysis and Time Window Selection}

Time-Amplitude analyses of the EEG recordings were performed for each sub-data set. The purpose of these analyses was to observe the amplitude of brain responses upon the presentation of the images and examine how these responses would change between the original and bright groups. For this purpose, the average amplitude of all the EEG epochs belonging to the 31 subjects in each sub-data set was calculated for each channel as in (3) and is shown in Fig. 2 in the results section for both the original and bright groups.

$$
X_{a a}=\frac{1}{N} \sum_{k=1}^{N} Y_{n_{k}}
$$

$$
W_{a p}=\left(X_{n a a}\right)^{2}
$$

where $X_{a a}$, denotes the average amplitude of any channel for the related emotional condition, $N$ is the total number of epochs of the 31 subjects and $W_{a p}$ is the average power of any channel for the related emotional condition.

\section{Time window selection}

After the average amplitude analysis of the EEG recordings, we attempted to determine time windows and channels, where changes in the power of brain responses were observed with brightness effect. For this purpose, we first calculated the average power of the EEG recordings for all the sub-data sets by using (4). Then, for each emotional condition, average power differences between the original and bright groups were calculated for 12 channels in the 0-1000 ms time window after the stimulus onset, as shown in Fig. 3. By using Fig. 3, time windows where brightness influenced the power of brain responses were determined as $T_{1}, T_{2}, T_{3}, T_{4}, T_{5}$ and $T_{6}$.

\subsubsection{Time-Frequency Analysis and Feature Extraction}

Time-frequency analysis methods are widely used in the investigation of EEG signals. With these methods, we can observe the frequency content of the signal at each time point of signal. In this study, the STFT was used as the time-frequency analysis method.

\section{Short time Fourier transform (STFT) method}

STFT is defined as (5) and is described as the discrete time Fourier transform evaluated on a moving window. The STFT power spectrogram is defined as (6), where $\mathrm{n}$ is the discrete-time index, $y(k)$ is an input signal, and $w(n-k)$ is a real-valued window function which describes the part of $y(n)$ used in the calculation of $Y(n, w)[49,50]$. 


$$
\begin{aligned}
& Y(n, w)=\sum_{k=-\infty}^{\infty} y(k) w(n-k) e^{-j w k} \\
& S_{y}(n, w)=|Y(n, w)|^{2}
\end{aligned}
$$

Various window functions are available in STFT. We compared the performances of different window functions to find the most suitable one for the study. Since we did not fully know the nature of EEG signals and we intended to use the softening window, the Hanning window was preferred as the window for the study. The Hanning window reduces spectral leakage. Moreover, it has a wide peak and narrow side lobes. The choice of the window size is important in STFT for the desired time and frequency resolution. We were interested in the frequency range of $0.5-70 \mathrm{~Hz}$ in the study and thus the appropriate window size was chosen as 128 sample lengths. The overlapping of the window was set to (window size - 1).

In the present study, brightness effect of visual stimuli was evaluated on the power of brain signals for different frequency bands. To this end, the average EEG power spectrogram of all the epochs belonging to the 31 subjects in each sub-data set was obtained separately for 12 channels using (6). These spectrograms showed the distribution of the power of the EEG recordings in the timefrequency plane. When the average EEG power spectrograms were examined for the specified time windows, differences were observed between the average power spectrograms of the original and bright groups on the specific frequency and channels. To determine the frequency bands where these differences were observed in each channel, the average EEG power spectrograms of the bright groups were subtracted from the average EEG power spectrograms of the original groups. The difference of the average EEG power spectrograms for each channel was shown in the time-frequency plane (Fig. 4). Frequency bands, in which brightness affected the power of brain responses, were examined through these spectrogram differences.

\section{Feature extraction}

In EEG studies, it is important to extract features that reflect the frequency, time and spatial characteristics of the signal. These features represent EEG signals. After observing brightness effect in the time and frequency plane analyses of the EEG recordings, in order to evaluate whether this effect was statistically significant, features were extracted from the average EEG power spectrograms and tested statistically. For this purpose, the power spectrogram of the average of all the EEG epochs in a single channel for each of the 31 subjects was obtained for 12 channels separately. The spectrograms were separated into predetermined time windows $\left(T_{1}, T_{2}, T_{3}, T_{4}\right.$ and $\left.T_{5}\right)$ and frequency bands (delta, theta, alpha, beta and gamma). The average power values per frequency and time were extracted at each frequency band for each of the defined time windows as features. These features were obtained for all the sub-data sets: Poriginalpleasant, Poriginalunpleasant, PORIGINALneutral, $\boldsymbol{P}_{\text {BRIGHTpleasant, }}$ $\boldsymbol{P}_{\text {BRIGHTunpleasant }} \boldsymbol{P}_{\text {BRIGHTneutral. }}$ The average power values were assessed in decibel (dB) units obtained with the $\log$ power transformation $\left(10 * \log _{10}\left(S_{y}\right)\right)$. Each feature size was $31 \times 12$ (subjects $\mathrm{x}$ channels). Feature extraction steps are shown as the flowchart in Fig. 1 . Then, the features were compared between the original and bright groups using statistical methods. Our goal here was to compare the average power values per frequency and time between the original and bright data groups and understand how brightness would change the power of responses. Moreover, channels, time and frequency ranges in which these changes were effective were also determined statistically. 


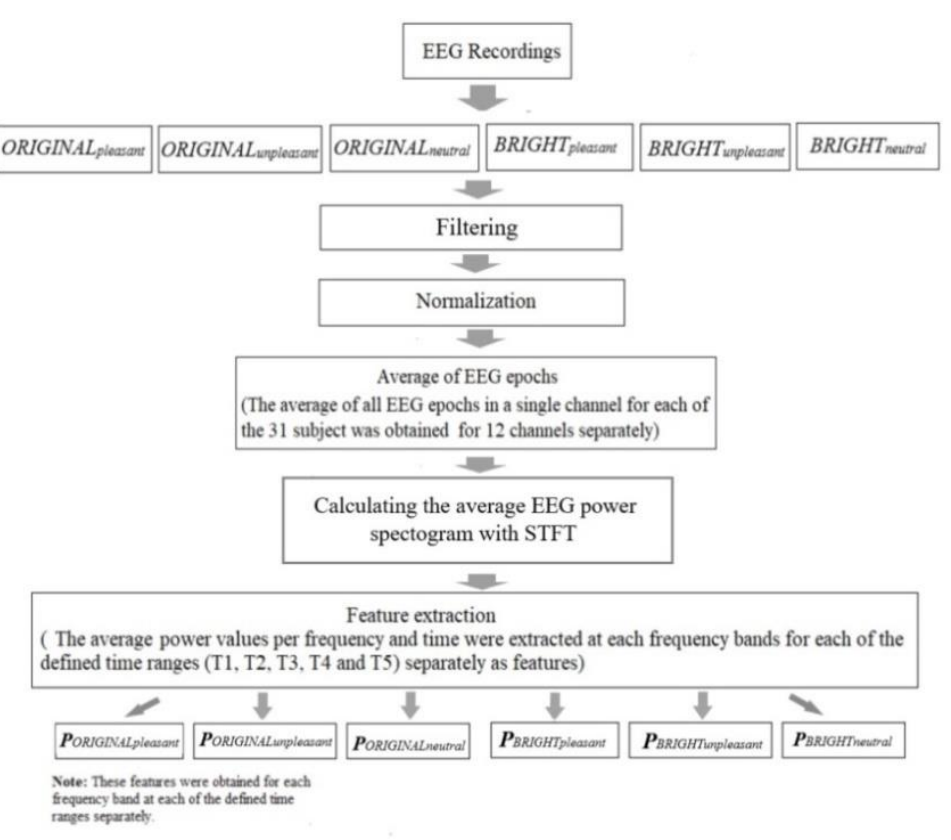

Figure 1. The flowchart of feature extraction.

\subsection{Statistical Analysis}

All statistical analyses were calculated using SPSS. The first statistical analysis was performed for EEG recordings. The repeated measures of ANOVA were performed to observe differences between the factors in the whole topology for features obtained from 12 channels of the 31 subjects. The repeated measures of ANOVA included the within-subject factors as locations (frontal, central, parietal, occipital, 4) x saggital (right, central, left, 3) and the between-subject factor as status (original, bright, 2). Mauchly' s test evaluated the sphericity assumption when necessary. Greenhouse-Geisser corrected p-values were reported. Differences in-group means were tested using independent samples $t$-tests with the Bonferroni test. The significance level was set to $\mathrm{p}<0.05$ for all comparisons. For each emotional condition, all the statistical analyses were repeated for features obtained from different time windows $\left(\mathrm{T}_{1}, \mathrm{~T}_{2}, \mathrm{~T}_{3}, \mathrm{~T}_{4}\right.$ and $\left.\mathrm{T}_{5}\right)$ of each frequency band (delta, theta, alpha, beta and gamma).

The second statistical analysis was performed for behavioral data. The result of valence and arousal SAM-ratings for the bright and original emotional images were compared usingthe one-way ANOVA test, status (original, bright, 2) $\mathrm{x}$ emotional conditions (pleasant, unpleasant, neutral, 3 ). Moreover, a t-test was used for post hoc comparison.

\section{Results}

In the following sub-sections, EEG data analysis results, statistical analysis results, and topological representation of results on scalp are presented.

\subsection{Results of Time-Amplitude Analysis of Electroencephalography (EEG) Data}

In Fig. 2, the first and second rows show the average amplitude graphs of all the EEG epochs belonging to the 31 subjects for each channel in the original and bright groups, respectively. In each graph, the vertical axis shows the average amplitude values and the horizontal axis shows time (ms). The vertical red line in each graph indicates the onset of the visual stimulus. The pink, cyan and black curves in the graphs represent brain responses from 12 channels obtained upon the presentation of the pleasant, unpleasant and neutral stimuli, respectively. As observed in the graphs, a marked increase was observed in brain activity after the stimulus compared to the pre-stimulus brain activity and was clearly reflected on the EEG. By comparing the amplitudes of responses in the brain after the presentation of visual stimuli between the original and bright groups for all three 
types of stimuli, noticeable amplitude differences is observed at the same periods of time for the both groups. This observation indicated that the brightness of visual stimuli was effective on the amplitude of brain responses. Therefore, this effect was reflected in the power of these responses.
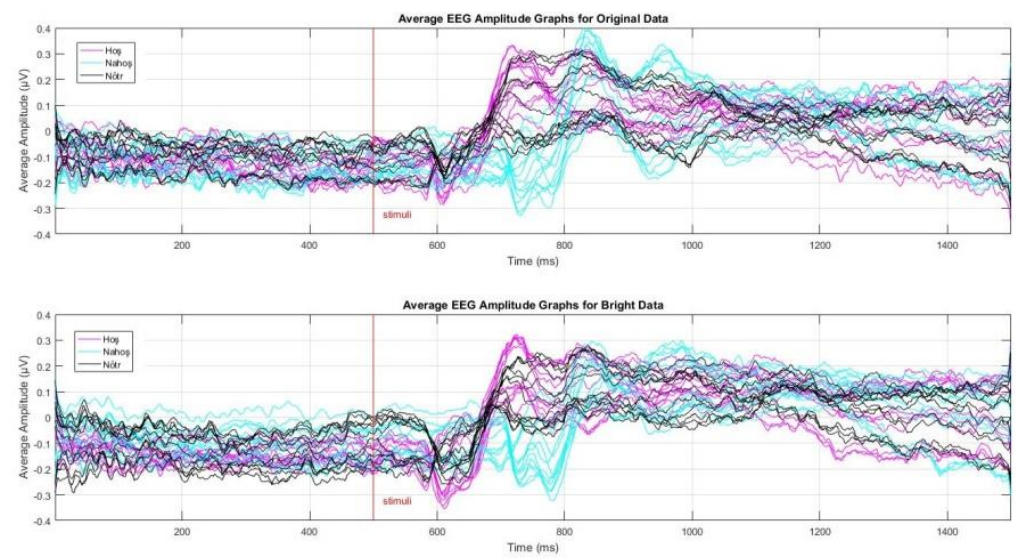

Figure 2. The average amplitude graphs of all the EEG epochs belonging to the 31 subjects for each channel in the original and bright groups; a) the average amplitude graphs of 12 channels for ORIGINALpleasant, ORIGINALunpleasant and ORIGINALneutral ; b) the average amplitude graphs of 12 channels for BRIGHT pleasant, BRIGHT unpleasant and BRIGHT neutral.

The average power differences between the original and bright groups were created to determine time windows, in which brightness effect of emotional visual stimuli would be observed on brain responses. In Fig. 3, the first, second and third rows show the average power differences for the pleasant, unpleasant and neutral conditions from 12 channels, respectively. In each graph, the vertical axis shows channels (top to bottom respectively: $\mathrm{F}_{3}, \mathrm{~F}_{2}, \mathrm{~F}_{4}, \mathrm{C}_{3}, \mathrm{C}_{2}, \mathrm{C}_{4}, \mathrm{P}_{3}, \mathrm{P}_{z}, \mathrm{P}_{4}, \mathrm{O}_{1}, \mathrm{O}_{2}$ and $\mathrm{O}_{2}$ ) and the horizontal axis shows time (1000 ms after the visual stimulus). The color scale, which expresses the difference of the average power values of the original and bright groups, appears on the right side of the figure.

It is evident that Fig. 3 offers the average power difference of the EEG recordings to be considered between the original and bright groups in specific time windows for each emotional condition. The areas where intense colors are observed in the graphs indicate channels and time windows, where brightness effect is apparent. According to Fig. 3, with the increase in the brightness of visual stimuli, the average power of brain responses decreased in the light colored areas but, increased in the dark colored areas. Time windows, in which brightness effect was observed intensely in the graphs, were determined as $\mathrm{T}_{1}=100-200 \mathrm{~ms}, \mathrm{~T}_{2}=200-300 \mathrm{~ms}, \mathrm{~T}_{3}=300-400 \mathrm{~ms}, \mathrm{~T}_{4}=400-$ $500 \mathrm{~ms}, \mathrm{~T}_{5}=500-600 \mathrm{~ms}$ and $\mathrm{T}_{6}=600-700 \mathrm{~ms}$. The time windows were obtained from the average values of the 31 subjects. In the following sections, it was statistically analyzed whether brightness was effective at these time windows for each person. The average power difference of the EEG recordings showed that in the early and late time windows of visual processing, brightness had different effects for different emotional states. 


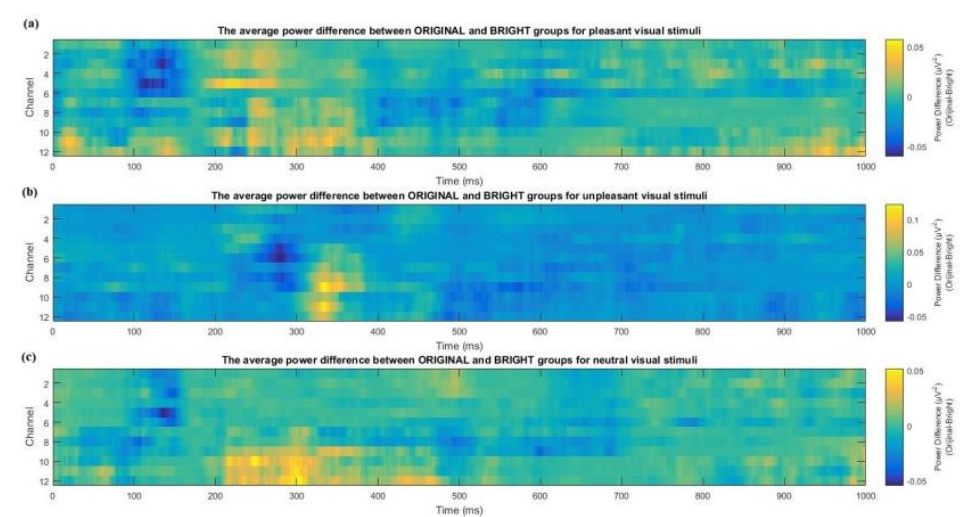

356 Figure 3. The average power differences between the original and bright groups for 12 channels in the $0-1000 \mathrm{~ms}$ time window after the visual stimulus onset; a) the average power differences between the original and bright groups for the the pleasant visual stimuli; b) the average power differences between the original and bright groups for the unpleasant visual stimuli; c) the average power differences between the original and bright groups for the neutral visual stimuli.

\subsection{Results of Time-Frequency Analysis of Electroencephalography (EEG) Data}

In Fig. 4, the difference of the average power spectrograms between the original and bright groups was shown on the time-frequency plane for 12-channels for the pleasant, unpleasant and neutral conditions. In each spectrogram, the vertical axis shows frequency $(0-70 \mathrm{~Hz})$ and the horizontal axis shows time ( $\left.\mathrm{T}_{1}-\mathrm{T}_{5}\right)$. The color scale, which expresses the difference values of the average power spectrograms belonging to original and bright groups, appears on the right side of the figures. Changes of brightness of visual stimuli affected the power of brain oscillation responses. Therefore, power differences were observed between the average power spectrograms of the original and bright groups. Areas where intense color differences were observed in the power difference spectrograms indicate time, frequency and channels where brightness effect is apparent. It is clearly shown in Fig. 4 that the responses of the brain to the change of brightness of visual stimuli change multidimensionally according to frequency band, time window, channel and emotional condition. At the end of the time-frequency analyses, frequency bands, in which the brightness effect of visual stimuli was investigated on brain responses, were determined as delta, theta, alpha, beta and gamma. In order to evaluate whether these findings were statistically significant, statistical analysis was performed on features extracted from each individual's average EEG power spectrogram. 

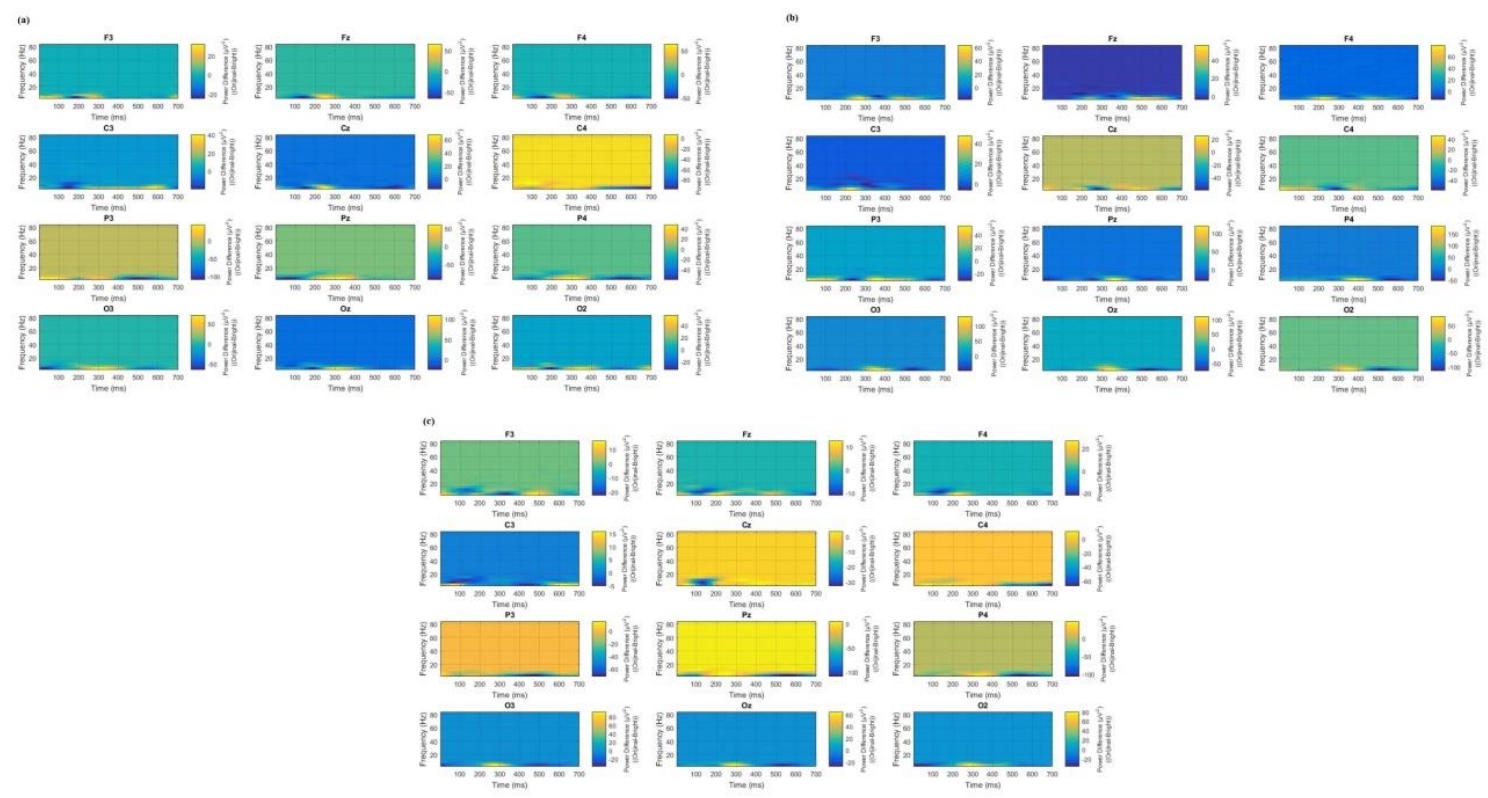

Fig. 4. The difference between the average EEG power spectrograms of the original and bright groups for 12 channels $\left(\mathrm{F}_{3}, \mathrm{~F}_{z}, \mathrm{~F}_{4}, \mathrm{C}_{3}, \mathrm{C}_{2}, \mathrm{C}_{4}, \mathrm{P}_{3}, \mathrm{P}_{z}, \mathrm{P}_{4}, \mathrm{O}_{1}, \mathrm{O}_{z}\right.$, and $\left.\mathrm{O}_{2}\right)$ in the time-frequency plane; a) the difference between the average EEG power spectrograms in the neutral visual stimuli; $b$ ) the difference between the average EEG power spectrograms in the unpleasant visual stimuli; c) the difference between the average EEG power spectrograms in the pleasant visual stimuli.

In power analyses performed as pre-evaluation, during visual processing, it was observed that the brightness of visual stimuli changed the power of the average brain responses of the 31 participants at certain time, frequency and electrode points. In the next section, it was statistically tested whether this observation was significant using features obtained from the EEG power spectrograms of each participant.

\subsection{Statistical Analysis Results}

The features obtained for the pleasant, unpleasant and neutral visual stimuli were compared statistically between the original and bright groups for different time windows, frequency bands and channels, and significant results are shown in Tables 2-4.

The repeated measures of ANOVA revealed significant results for only the location $x$ status effect between the original and bright status of the pleasant, unpleasant and neutral emotional stimuli in different bands and time windows. In other words, statistically significant effects of brightness were observed on location.

\subsubsection{Statistical Analysis Results for Pleasant Stimuli}

For delta band, there was no significant result for unpleasant stimuli. For theta band, in T2 (200$300 \mathrm{~ms}), \mathrm{T} 5(500-600 \mathrm{~ms})$ and T6 (600-700 ms) time windows, respectively $(\mathrm{F}(2.54,152.31)=4.25, \mathrm{p}=0.01$, $\mathrm{F}(2.30,137.85)=3.27, \mathrm{p}=0.03$ and $\mathrm{F}(2.30,137.85)=3.27, \mathrm{p}=0.03)$, for alpha band, in $\mathrm{T} 2$ (200-300 ms), $\mathrm{T} 5(500-600 \mathrm{~ms})$ and $\mathrm{T} 6(600-700 \mathrm{~ms})$ time windows, respectively $(\mathrm{F}(2.11,126.75)=3.58, \mathrm{p}=0.02$, $\mathrm{F}(2.27,136.16)=3.32$, $\mathrm{p}=0.03$ and $\mathrm{F}(2.27,136.16)=3.32, \mathrm{p}=0.03)$, for beta band, in T2 (200-300 ms), T3(300$400 \mathrm{~ms}), \mathrm{T} 4(400-500 \mathrm{~ms}), \mathrm{T} 5(500-600 \mathrm{~ms})$ and T6 $(600-700 \mathrm{~ms})$ time windows, respectively $(\mathrm{F}(2.61,156.60)=7.52, \quad \mathrm{p}=0.00, \quad \mathrm{~F}(2.50,149.99)=7.70, \quad \mathrm{p}=0.00, \quad \mathrm{~F}(2.27,136.25)=6.63, \quad \mathrm{p}=0.00$, $\mathrm{F}(2.30,137.74)=4.41, \mathrm{p}=0.01$ and $\mathrm{F}(2.30,137.74)=4.41, \mathrm{p}=0.01)$ and for gamma band, in $\mathrm{T} 1(100-200 \mathrm{~ms})$, T2 (200-300 ms), T3(300-400 ms), T4(400-500 ms), T5(500-600 ms) and T6 (600-700 ms) time 
406 windows, respectively $(\mathrm{F}(2.54,152.46)=8.84, \mathrm{p}=0.00, \mathrm{~F}(2.41,146.66)=8.76, \mathrm{p}=0.00, \mathrm{~F}(2.29,137.18)=7.22$, $407 \mathrm{p}=0.00, \mathrm{~F}(2.27,136.50)=6.73, \mathrm{p}=0.00, \mathrm{~F}(2.36,141.77)=10.24, \mathrm{p}=0.00$ and $\mathrm{F}(2.36,141.77)=10.24, \mathrm{p}=0.00)$, 408 significant results were observed.

409 The independent t-test was performed to compare the means of features and determine electrode 410 sites where significant differences were observed. The electrode sites, group means and 411 corresponding statistical analysis values, in which significant differences were observed for the 412 specified time windows and frequency bands, are summarized in Table 2. 
13 of 29

Table 2. The summary of the time windows $\left(T_{1}, T_{2}, T_{3}, T_{4}, T_{5}\right.$ and $\left.T_{6}\right)$, frequency bands (delta, theta, alpha, beta and gamma) and electrode sites, where statistically significant differences $(\mathrm{p}$-value $<0.05)$ in the average log powers were observed between the original and bright groups as features, for pleasant stimuli according to the independent $t$-test results; The statistical parameters, group mean $\left(\boldsymbol{m e a n}_{\text {original }} \boldsymbol{m e a n}_{\text {bright }}\right)$ and standard deviation $\left(\boldsymbol{s t d}_{\text {original }}, \boldsymbol{s t d}_{\text {bright }}\right)$ of features belonging to the 31 subjects for the original and bright groups.

\begin{tabular}{|c|c|c|c|c|c|c|}
\hline \multicolumn{7}{|c|}{ Electrode Site: mean $_{\text {original }}\left(\right.$ std $\left._{\text {original }}\right)$, mean $_{\text {bright }}\left(\right.$ std $\left._{\text {bright }}\right)$, p-value } \\
\hline & T1 (100-200 ms) & T2 (200-300 ms) & T3 $(300-400 \mathrm{~ms})$ & $\mathrm{T} 4(400-500 \mathrm{~ms})$ & T5 $(500-600 \mathrm{~ms})$ & T6 $(600-700 \mathrm{~ms})$ \\
\hline 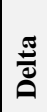 & -- & -- & -- & -- & -- & -- \\
\hline & -- & $\begin{array}{l}\mathbf{F}_{3}: 18.29(2.63), 15.45(2.74), .00 \\
\mathbf{F}_{\mathbf{Z}}: 19.10(3.43), 16.51(3.69), .01 \\
\mathbf{C}_{3}: 17.37(3.56), 15.30(2.57), .01 \\
\left.\mathbf{C}_{\mathbf{Z}}: 19.22(3.00), 16.603 .20\right), .00\end{array}$ & -- & -- & $\begin{array}{l}\mathbf{C}_{3}: 16.98(3.53), 13.88(3.74), .00 \\
\mathbf{C}_{\mathbf{Z}}: 16.66(4.74), 13.73(3.68), .01\end{array}$ & $\begin{array}{l}\mathbf{C}_{3}: 16.98(3.52), 13.88(3.74), .00 \\
\mathbf{C}_{\mathbf{Z}}: 16.66(4.74), 13.73(3.68), .01\end{array}$ \\
\hline$\frac{\pi}{\frac{\pi}{2}}$ & -- & $\begin{array}{l}\mathbf{C}_{3}: 14.26(4.30), 11.56(3.03), .01 \\
\mathbf{P}_{4}: 10.71(5.18), 13.48(4.78), .03\end{array}$ & -- & -- & $\begin{array}{l}\mathbf{C}_{3}: 11.19(4.40), 8.51(5.36), .03 \\
\mathbf{C}_{\mathbf{Z}}: 10.89(4.24), 8.23(3.66), .01\end{array}$ & $\begin{array}{l}\mathbf{C}_{3}: 11.19(4.40), 8.52(5.36), .03 \\
\mathbf{C}_{\mathbf{Z}}: 10.89(4.24), 8.23(3.66), .01\end{array}$ \\
\hline صّ & -- & $\begin{array}{l}\mathbf{F}_{\mathbf{Z}}: 4.37(4.03), 2.36(3.25), .03 \\
\mathbf{C}_{\mathbf{3}}: 5.43(4.29), 2.21(3.13), .00 \\
\mathbf{C}_{\mathbf{Z}}: 4.27(3.82), 1.72(1.82), .00 \\
\mathbf{O}_{\mathbf{1}}: 0.39(3.49), 2.54(3.40), .02\end{array}$ & $\begin{array}{l}\mathbf{F}_{3}: 3.79(4.28), 0.27(3.57), .00 \\
\mathbf{F}_{\mathbf{Z}}: 3.52(3.51), 1.32(3.93), .02 \\
\left.\mathbf{F}_{\mathbf{4}}: 4.54(3.22), 2.084 .27\right), .01 \\
\mathbf{C}_{3}: 5.03(4.00), 1.98(3.31), .00 \\
\mathbf{C}_{\mathbf{Z}}: 3.54(3.67), 1.45(2.51), .00 \\
\mathbf{O}_{\mathbf{1}}:-0.29(3.32), 1.72(3.41), .01\end{array}$ & $\begin{array}{l}\mathbf{F}_{3}:-3.87(3.53), 1.56(3.84), .02 \\
\mathbf{F}_{\mathbf{Z}}: 3.33(3.05), 1.11(3.66), .01 \\
\mathbf{F}_{\mathbf{4}}: 3.66(3.67), 1.76(3.35), .03 \\
\mathbf{C}_{\mathbf{3}}: 4.94(3.95), 1.75(4.01), .00 \\
\mathbf{C}_{\mathbf{Z}}: 3.47(3.82), 1.50(2.98), 03\end{array}$ & $\begin{array}{l}\mathbf{F}_{3}: 3.75(3.73), 0.58(4.65), .01 \\
\mathbf{F}_{\mathbf{Z}}: 3.17(3.88), 0.57(3.56), .01 \\
\mathbf{C}_{3}: 5.81(4.20), 0.93(4.55), .00 \\
\mathbf{C}_{\mathbf{Z}}: 3.76(3.43), 0.90(2.88), .00\end{array}$ & $\begin{array}{l}\mathbf{F}_{3}: 3.75(3.73), 0.58(4.65), .00 \\
\mathbf{F}_{\mathbf{Z}}: 3.17(3.88), 0.57(3.56), .01 \\
\mathbf{C}_{3}: 3.81(4.20), 0.93(4.55), .00 \\
\mathbf{C}_{\mathbf{Z}}: 3.76(3.43), 0.90(2.88), .00\end{array}$ \\
\hline שَّ & $\begin{array}{l}\mathbf{F}_{3}:-4.37(4.07),-7.84(2.86), .00 \\
\mathbf{F}_{\mathbf{Z}}:-5.31(2.83),-7.31(2.85), .01 \\
\mathbf{F}_{\mathbf{4}}:-3.61(2.92),-6.21(3.47), .01 \\
\mathbf{C}_{3}:-18.14(4.52),-20.76(3.11), .00 \\
\mathbf{C}_{\mathbf{Z}}:-4.86(3.43),-6.89(2.43), .01\end{array}$ & $\begin{array}{l}\mathbf{F}_{3}:--4.19(3.73),-75(3.59), .00 \\
\mathbf{F}_{\mathbf{Z}}:-5.00(3.60),-7.18(2.96), .01 \\
\mathbf{F}_{\mathbf{4}}:-5.51(3.96),-6.15(3.77), .01 \\
\mathbf{C}_{3}:-3.13(4.84),-6.01(3.24), .01 \\
\mathbf{C}_{\mathbf{Z}}:-4.54(3.39),-6.60(3.70), .02\end{array}$ & $\begin{array}{l}\mathbf{F}_{3}:-4.22(3.68),-7.23(3.53), .00 \\
\mathbf{F}_{4}:-3.99(3.80),-6.21(3.71), .02\end{array}$ & $\begin{array}{l}\mathbf{F}_{3}:-4.33(3.75),-7.98(2.65), .00 \\
\mathbf{F}_{4}:-4.19(4.23),-6.55(3.66), .02 \\
\mathbf{C}_{3}:-3.92(4.16),-6.92(3.71), .00 \\
\mathbf{C}_{\mathbf{Z}}:-5.57(3.26),-7.74(3.06), .01\end{array}$ & $\begin{array}{l}\mathbf{F}_{3}:-4.12(4.36),-7.87(2.68), .00 \\
\mathbf{F}_{4}:-3.75(3.91),-6.10(3.28), .01 \\
\mathbf{C}_{3}:-3.34(4.21),-6.82(3.57), .00 \\
\mathbf{C}_{\mathbf{Z}}:-5.38(2.89),-7.67(2.91), .00 \\
\mathbf{O}_{1}:-7.67(3.36),-5.82(5.59), .04\end{array}$ & $\begin{array}{l}\mathbf{F}_{3}:-4.12(4.36),-7.87(2.68), .00 \\
\mathbf{F}_{4}:-3.75(3.91),-6.10(3.28), .01 \\
\mathbf{C}_{3}:-3.34(4.21),-6.82(3.57), .00 \\
\mathbf{C}_{\mathbf{Z}}:-5.38(2.89),-7.67(2.91), .03 \\
\mathbf{O}_{1}:-7.67(3.36),-5.82(3.59), .04\end{array}$ \\
\hline
\end{tabular}




\section{$1 \quad$ 3.3.1. Statistical analysis results for unpleasant stimuli}

For delta band, in $\mathrm{T}_{1}(100-200 \mathrm{~ms})$ and $\mathrm{T}_{4}(400-500 \mathrm{~ms})$ time windows, respectively $3 \quad(\mathrm{~F}(2.61,156.76)=4.93, \mathrm{p}=0.00$ and $\mathrm{F}(2.63,157.73)=3.4, \mathrm{p}=0.02)$, for theta band, in $\mathrm{T}_{1}(100-200 \mathrm{~ms})$ and $\mathrm{T}_{4}$ 4 (400-500 ms) time windows, respectively $(F(3,180)=4.99, p=0.00$ and $F(3,180)=4.91, p=0.00)$, for alpha 5 band, in $\mathrm{T}_{3}(300-400 \mathrm{~ms})$ and $\mathrm{T}_{4}(400-500 \mathrm{~ms})$ time windows, respectively $(\mathrm{F}(2.55,153.23)=7.25 \mathrm{p}=0.04$ 6 and $\mathrm{F}(3,180)=3.17, \mathrm{p}=0.03)$,for beta band, in $\mathrm{T}_{1}(150-200 \mathrm{~ms}), \mathrm{T}_{2}(200-300 \mathrm{~ms})$ and $\mathrm{T}_{3}(200-400 \mathrm{~ms})$ time 7 windows, respectively $(\mathrm{F}(3,180)=3.31, \mathrm{p}=0.02, \mathrm{~F}(2.33,140.23)=3.69, \mathrm{p}=0.01$ and $\mathrm{F}(2.59,155.29)=4.25$, $8 \mathrm{p}=0.01)$ and for gamma band, in $\mathrm{T}_{3}(300-400 \mathrm{~ms}), \mathrm{T}_{4}(400-500 \mathrm{~ms}), \mathrm{T}_{5}(500-600 \mathrm{~ms})$ and $\mathrm{T}_{6}(600-700 \mathrm{~ms})$ 9 time windows, respectively $(\mathrm{F}(3,180)=3.48, \mathrm{p}=0.02, \mathrm{~F}(2.69,161.26)=3.47, \mathrm{p}=0.02, \mathrm{~F}(3,180)=4.87, \mathrm{p}=0.00$ 10 and $\mathrm{F}(3,180)=4.87, \mathrm{p}=0.00)$, significant results were observed. The independent $t$-test was performed 11 to compare the means of features and determine electrode sites where significant differences weres 12 observed. The electrode sites, group means and corresponding statistical analysis values, in which 13 significant differences were observed for the specified time windows and frequency bands, are 14 summarized in Table 3. 
Table 3. The summary of the time windows $\left(\mathrm{T}_{1}, \mathrm{~T}_{2}, \mathrm{~T}_{3}, \mathrm{~T}_{4}, \mathrm{~T}_{5}\right.$ and $\mathrm{T}_{6}$ ), frequency bands (delta, theta, alpha, beta and gamma) and electrode sites, where statistically significant differences $(\mathrm{p}$-value $<0.05)$ in the average log powers were observed between the original and bright groups as features, for unpleasant stimuli according to the independent $t$-test results; The statistical parameters, group mean $\left(\boldsymbol{m e a n}_{\text {original }}, \boldsymbol{m e a n}_{\text {bright }}\right)$ and standard deviation $\left(\boldsymbol{s t d}_{\text {original }}, \boldsymbol{s t d}_{\text {bright }}\right)$ of features belonging to the 31 subjects for the original and bright groups.

\begin{tabular}{|c|c|c|c|c|c|c|}
\hline \multicolumn{7}{|c|}{ Electrode Site: mean $_{\text {original }}\left(\right.$ std $\left._{\text {original }}\right)$, mean $_{\text {bright }}\left(\right.$ std $\left._{\text {bright }}\right)$, p-value } \\
\hline & $T_{1}(100-200 \mathrm{~ms})$ & $T_{2}(200-300 \mathrm{~ms})$ & $\mathrm{T}_{3}(\mathbf{3 0 0 - 4 0 0 \mathrm { ms } )}$ & $\mathrm{T}_{4}(400-500 \mathrm{~ms})$ & $T_{5}(500-600 \mathrm{~ms})$ & $T_{6}(600-700 \mathrm{~ms})$ \\
\hline 苞 & $\mathrm{C}_{\mathbf{Z}}: 16.57(3.97), 14.15(3.99), .02$ & -- & -- & $\begin{array}{l}\mathbf{F}_{3}: 15.62(4.21), 12.96(4.14), .01 \\
\mathbf{C}_{3}: 15.73(3.94), 12.23(3.49), .00 \\
\mathbf{C}_{\mathbf{Z}}: 16.69(3.73), 14.70(3.26), .02 \\
\mathbf{C}_{4}: 15.90(3.55), 12.72(4.34), .02\end{array}$ & -- & -- \\
\hline ڤึّ & $\mathrm{F}_{\mathrm{Z}}:-0.29(3.18), 1.81(3.07), .00$ & $\mathrm{~F}_{\mathbf{Z}}: 1.56(3.14), 3.88(2.86), .00$ & $\begin{array}{l}\mathrm{C}_{\mathrm{Z}}: 4.75(3.56), 2.31(3.11), .01 \\
\mathrm{C}_{4}: 5.19(3.48), 2.03(3.62), .00\end{array}$ & -- & -- & -- \\
\hline
\end{tabular}




\section{$1 \quad$ 3.3.2. Statistical analysis results for neutral stimuli}

For delta band, in $\mathrm{T}_{3}(300-400 \mathrm{~ms})$ and $\mathrm{T}_{4}(400-500 \mathrm{~ms})$ time windows, respectively $(\mathrm{F}(2.47,143.08)=3.60, \mathrm{p}=0.02, \mathrm{~F}(2.50,145.28)=3.88, \mathrm{p}=0.01)$ and $\mathrm{F}(2.49,144.64)=3.47, \mathrm{p}=0.02)$, for theta band, in $\mathrm{T}_{3}(300-400 \mathrm{~ms})$, and $\mathrm{T}_{4}(400-500 \mathrm{~ms})$ time windows, respectively $(\mathrm{F}(2.37,137.66)=5.13, \mathrm{p}=0.00$, $\mathrm{F}(2.35,136.11)=4.48, \mathrm{p}=0.01)$, for alpha band, in $\mathrm{T}_{3}(300-400 \mathrm{~ms})$, and $\mathrm{T}_{4}(400-500 \mathrm{~ms})$ time windows, respectively $(\mathrm{F}(2.48,143.97)=3.29, \mathrm{p}=0.03$ and $\mathrm{F}(2.34,135.53)=3.76, \mathrm{p}=0.02)$, for gamma band, in $\mathrm{T}_{2}(200$ $300 \mathrm{~ms}), \mathrm{T}_{4}(300-400)$ and $\mathrm{T}_{6}(600-700)$ time windows, respectively $(\mathrm{F}(2.23,129.25)=3.96, \mathrm{p}=0.02$, $8 \mathrm{~F}(2.15,124.99)=3.25, \mathrm{p}=0.03$ and $\mathrm{F}(2.92,132.96)=3.45, \mathrm{p}=0.03)$, significant results were observed. But there was no significant result for alpha and gamma bands. The independent $t$-test was performed 10 determine the electrode sites where significant differences were observed. The electrode sites, group 11 means and corresponding statistical analysis values, in which significant differences were observed 12 for the specified time windows in the delta bands, are summarized in Table 4. 
24 Table 4. The summary of the time windows $\left(\mathrm{T}_{2}, \mathrm{~T}_{3}, \mathrm{~T}_{4}\right.$ and $\mathrm{T}_{6}$ ), frequency bands (delta, theta, alpha and gamma) and electrode sites, where statistically significant 25 differences ( $p$-value $<0.05$ ) in the average log powers were observed between the original and bright groups as features, for neutral stimuli according to the 26 independent $t$-test results; The statistical parameters, group mean $\left(\boldsymbol{m e a n}_{\text {original }}, \boldsymbol{m e a n}_{\text {bright }}\right)$ and standard deviation $\left(\boldsymbol{s t d}_{\text {original }}, \boldsymbol{s t d}_{\text {bright }}\right)$ of features belonging to the 2731 subjects for the original and bright groups.

\begin{tabular}{|c|c|c|c|c|}
\hline \multicolumn{5}{|c|}{ Electrode Site: mean $_{\text {original }}\left(\right.$ std $\left._{\text {original }}\right)$, mean $_{\text {bright }}\left(\right.$ std $\left._{\text {bright }}\right), \mathrm{p}$-value } \\
\hline & $T_{2}(200-300 \mathrm{~ms})$ & $T_{3}(300-400 \mathrm{~ms})$ & $T_{4}(400-500 \mathrm{~ms})$ & $T_{6}(600-700 \mathrm{~ms})$ \\
\hline \multirow{2}{*}{ 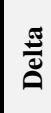 } & -- & & $\mathbf{C}_{\mathbf{4}}: 18.26(3.32), 15.46(6.04), .03$ & $\mathbf{P}_{\mathbf{Z}}: 11.83(6.02), 16.91(5.31)$ \\
\hline & & $P_{\mathrm{Z}}: 16.91(6.66), 20.27(4.37), .02$ & $2 \mathbf{P}_{\mathrm{Z}}: 16.12(6.20), 20.49(5.90), .02$ & $\mathbf{P}_{4}: 12.35(5.90), 16.26(5.30)$ \\
\hline \multirow{3}{*}{ 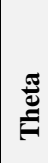 } & -- & $F_{3}: 16.74(3.06), 14.27(3.85), .01$ & $\mathbf{P}_{\mathrm{Z}}: 12.90(4.28), 15.95(4.51), .01$ & -- \\
\hline & & $F_{\mathrm{Z}}: 16.56(3.31), 13.96(3.93), .01$ & & \\
\hline & & $\mathrm{C}_{\mathbf{z}}: 16.04(3.27), 14.15(3.67), .04$ & & \\
\hline \multirow{2}{*}{$\frac{\pi}{2}$} & -- & $F_{3}: 11.31(3.50), 8.55(3.89), .00$ & $F_{Z}: 10.67(4.24), 7.96(3.95), .01$ & -- \\
\hline & & $F_{Z}: 11.52(4.72), 8.94(4.44), 03$ & & \\
\hline \multirow{3}{*}{ 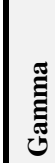 } & $\mathbf{P}_{\mathbf{Z}}:-7.84(3.22),-5.65(3.99), .02$ & \multirow[t]{3}{*}{--} & Pz: $-9.09(2.33),-7.18(3.77), .02$ & \multirow[t]{3}{*}{$\mathbf{P}_{\mathbf{Z}}:-9.05(3.15),-6.92(3.94), .02$} \\
\hline & $\mathbf{P}_{4}:-8.07(4.34),-5.95(3.27), .03$ & & $\mathbf{O}_{2}:-9.00(3.68),-7.04(3.43), .03$ & \\
\hline & $\mathbf{O}_{2}:-7.69(3.59),-5.70(3.40), .03$ & & & \\
\hline
\end{tabular}




\subsection{Topological Representation of Results on Scalp}

By using the results shown in Tables 2-4, Figs. 5-7 were created. As shown in the figures brightness effect on different time windows, frequency bands and channels appeared clearer and more comprehensive. Each large circle in the figures was created to represent the placement of 12 scalp electrode site was given on small circles within each large circle. In the figures, time transitions $\left(T_{1}, T_{2}, T_{3}, T_{4}, T_{5}\right.$ and $\left.T_{6}\right)$ were located from left to right and frequency band transitions (delta, theta, alpha, beta and gamma) were located from top to bottom in rows and columns. Electrode sites, where brightness effect was observed (according to Tables 2-4), were colored for each frequency band and time window. Blue color was used for electrode sites where the feature values were statistically significantly greater in original stimuli than in bright ones. Moreover, yellow color was used for electrode sites where the feature values were statistically significantly greater in bright stimuli than in original ones.

In Fig. 5, it is observed that brightness effect of the pleasant images on the electrophysiological responses started at the early time window $\left(\mathrm{T}_{1}=100-200 \mathrm{~ms}\right)$ and continued until the late time window $\left(\mathrm{T}_{6}=600-700 \mathrm{~ms}\right)$. This effect began to occur at high frequencies (gamma) in early time and was spread over a wide frequency range (beta, alpha and theta) over time. According to Fig. 5, the increase in the brightness of the pleasant images caused a decrease in the average power of gamma responses with short latency $\left(\mathrm{T}_{1}=100-200 \mathrm{~ms}\right)$ at the frontal and central regions $\left(\mathrm{F}_{3}, \mathrm{~F}_{2}, \mathrm{~F}_{4}, \mathrm{C}_{3}\right.$ and $\mathrm{C}_{\mathrm{z}}$ ). In the course of time, brightness also affected the occipital and parietal regions $\left(\mathrm{O}_{1}\right.$ and $\left.\mathrm{P}_{4}\right)$ parallel to the frontal and central regions $\left(\mathrm{F}_{3}, \mathrm{~F}_{2}, \mathrm{~F}_{4}, \mathrm{C}_{3}\right.$ and $\left.\mathrm{C}_{2}\right)$. The increase in the brightness of the pleasant images caused an increase in the average power of oscillational brain responses in the occipital and parietal regions, in contrast to the frontal and central regions.

In Fig. 6, it is observed that the brightness effect of the unpleasant images on the electrophysiological responses started at the early time window $\left(\mathrm{T}_{1}=100-200 \mathrm{~ms}\right)$ and continued until the $\mathrm{T}_{6}(600-700 \mathrm{~ms})$ time window. This effect began to occur at beta frequency in early time window and continued at relatively high frequencies (beta, alpha and gamma). Brightness effect was also spread over towards low frequencies (delta, theta) within the $\mathrm{T}_{4}(400-500 \mathrm{~ms}$ ) time window. According to Fig. 6, the increase in the brightness of the unpleasant images caused an increase in the average power of beta responses with short and middle latency $(100-300 \mathrm{~ms})$ at the frontal regions. In the course of time, brightness effect also continued in the frontal, central, occipital and parietal regions $\left(\mathrm{F}_{3}, \mathrm{C}_{3}, \mathrm{C}_{2}, \mathrm{C}_{4}, \mathrm{P}_{3}\right.$ and $\mathrm{Oz}$ ). Unlike other visual stimuli, for the unpleasant images, the increase in brightness caused a decrease in the average power of oscillational brain responses in the occipital and parietal regions. Another remarkable point is that, in contrast to other types of visual stimuli, the increase in the brightness of the unpleasant images caused an increase in the average power of brain activity in the early and middle time windows (100-300 ms) at the frontal regions.

In Fig. 7, it is observed that the brightness effect of the neutral images on the electrophysiological responses started later $\left(\mathrm{T}_{1}=200-300 \mathrm{~ms}\right)$ than other stimulus types and continued until the late time window $\left(\mathrm{T}_{6}=600-700 \mathrm{~ms}\right.$ ). However, no effect of luminance was observed within the $\mathrm{T}_{5}$ time window. As observed in the pleasant stimuli, the increase in the brightness of the neutral stimuli increased the average power of brain activity in the parietal and occipital regions, whereas decreased the power of brain activity in the frontal and central regions. Furthermore, unlike other types of visual stimuli, brightness effect for the neutral images was not observed in the beta band. 


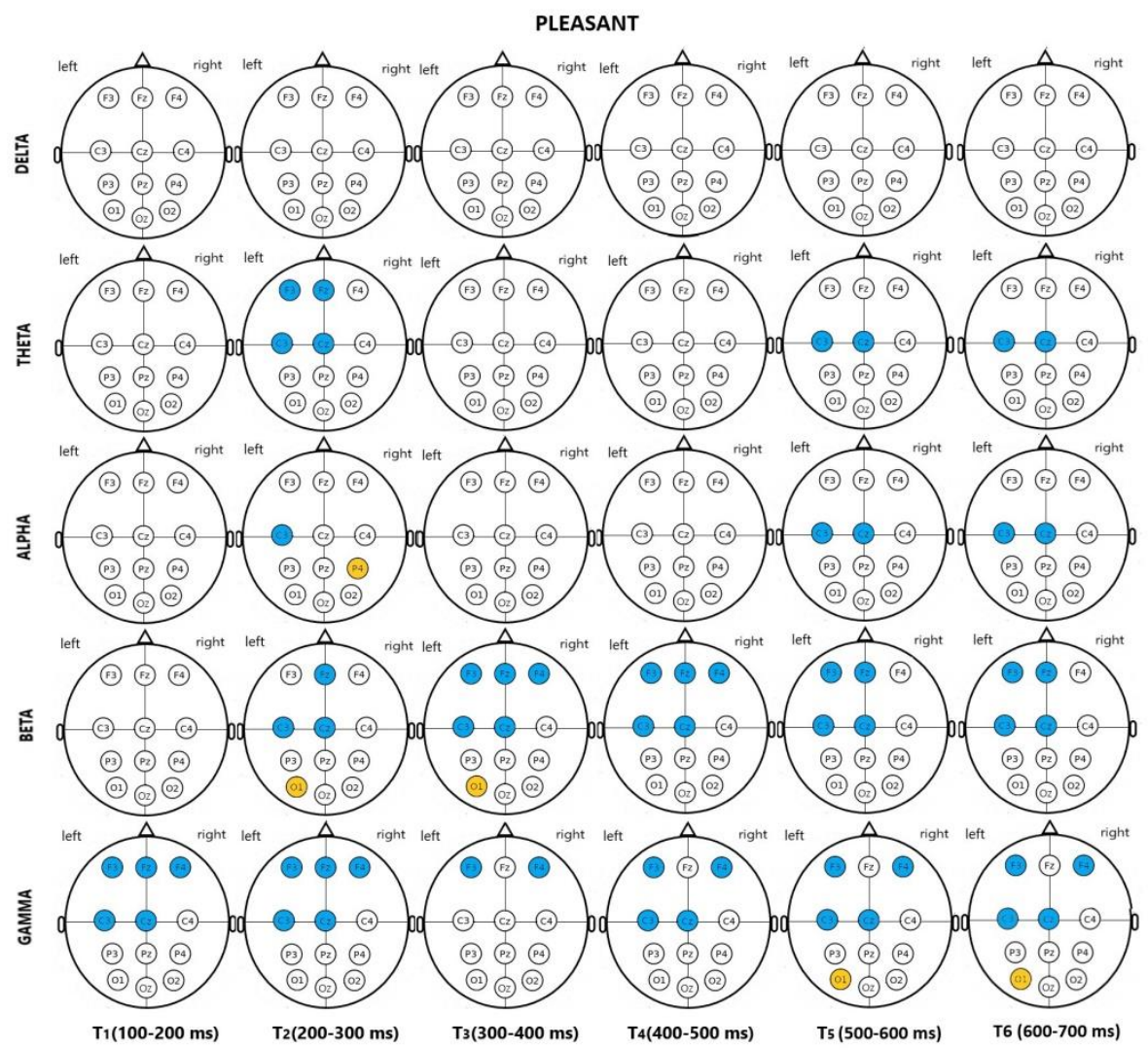

Figure 5. The electrode sites, where brightness effect is observed for different time windows and different frequency bands for pleasant stimuli. Blue color was used for electrode sites where the feature values were statistically significantly greater in the original stimuli than in the bright ones $(p<0.05)$. Yellow color was used for electrode sites where the feature values were statistically significantly greater in the bright stimuli than in the original ones. 


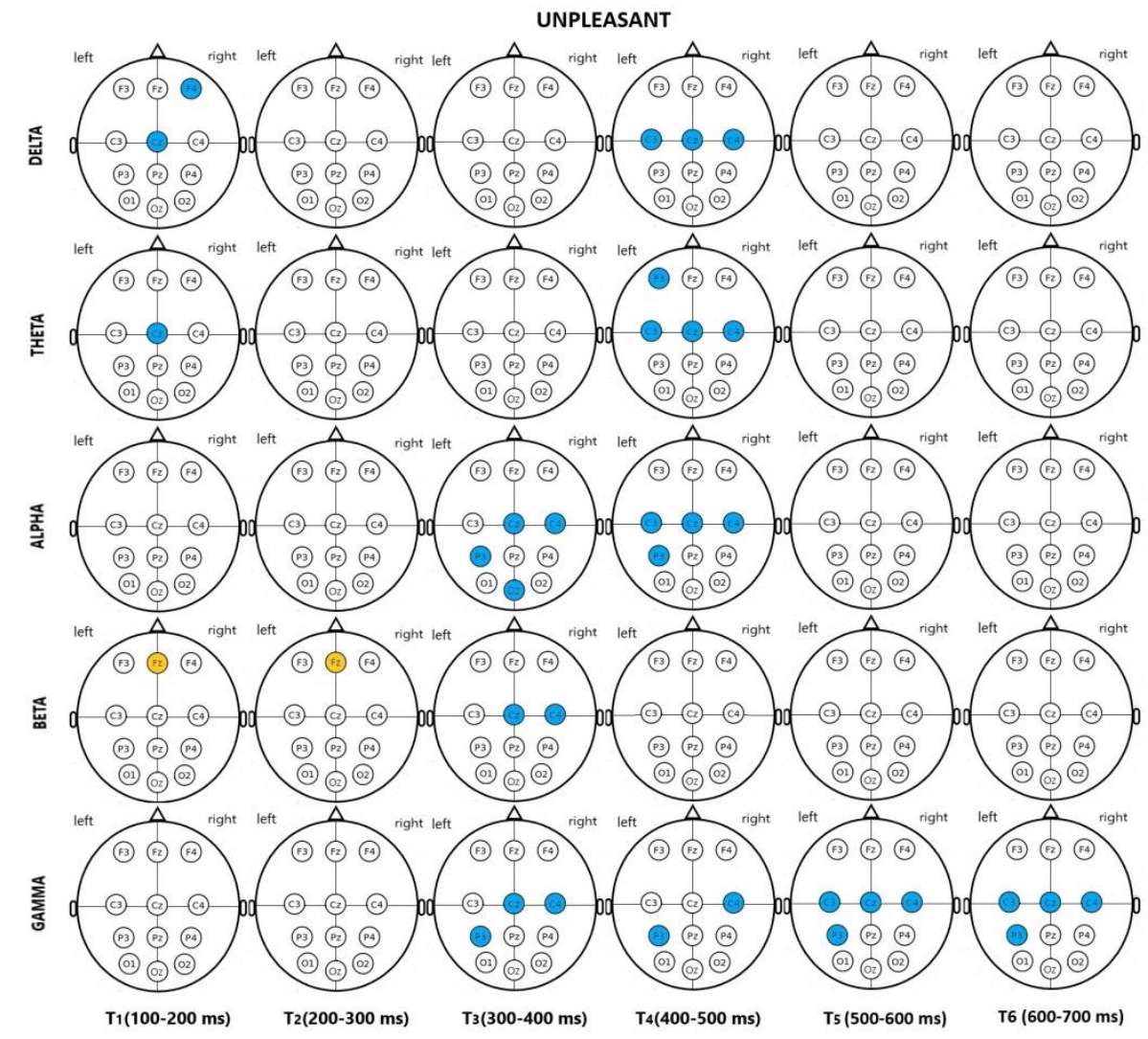

83 Figure 6. The electrode sites, where brightness effect is observed for different time windows and 84 different frequency bands for unpleasant stimuli. Blue color was used for electrode sites where the 85 feature values were statistically significantly greater in the original stimuli than in the bright ones 86 ( $p<0.05$ ). Yellow color was used for electrode sites where the feature values were statistically significantly greater in the bright stimuli than in the original ones. 


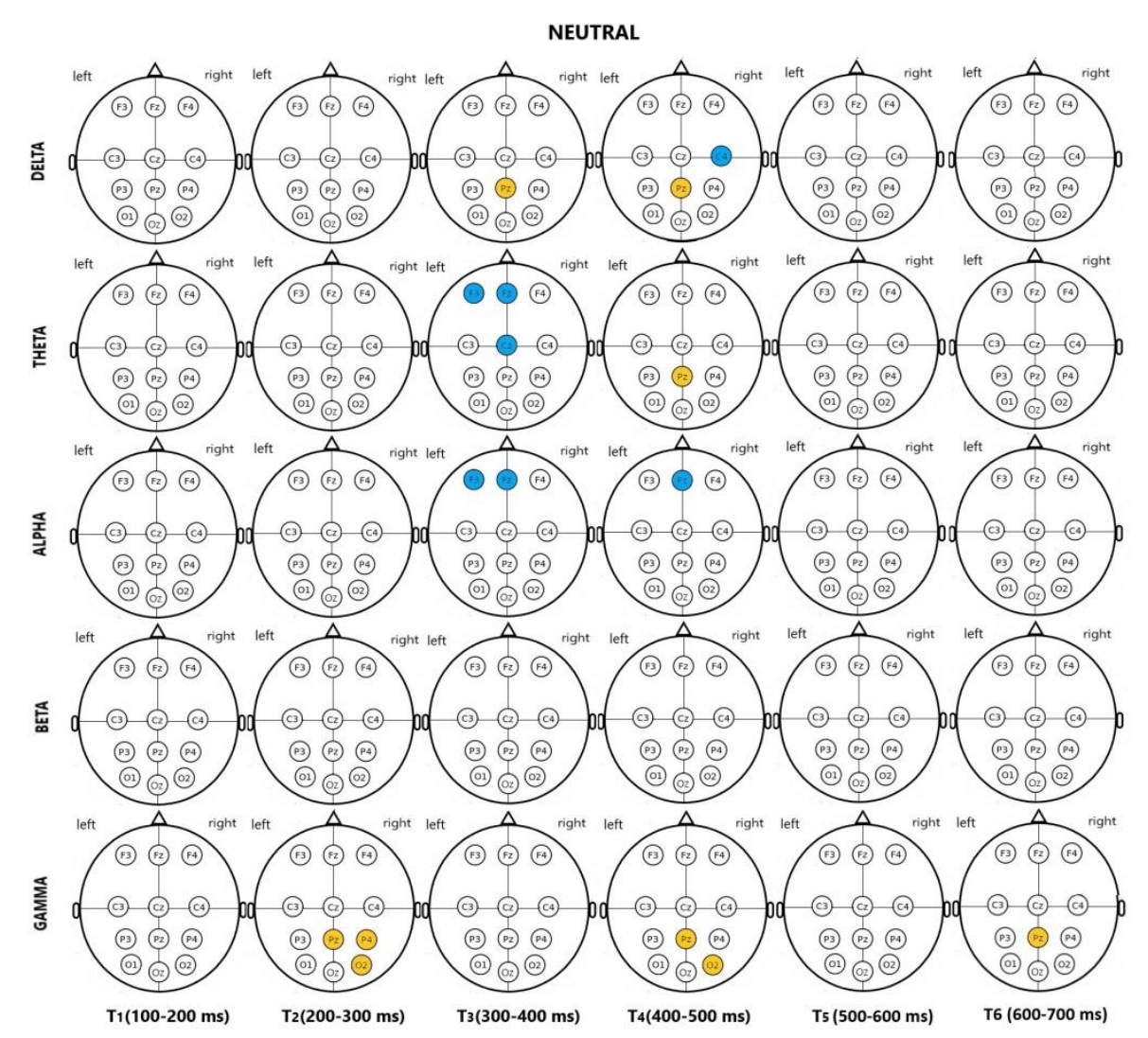

Figure 7. The electrode sites, where brightness effect is observed for different time windows and different frequency bands for neutral stimuli. Blue color was used for electrode sites where the feature values were statistically significantly greater in the original stimuli than in the bright ones $(\mathrm{p}<0.05)$. Yellow color was used for electrode sites where the feature values were statistically significantly greater in the bright stimuli than in the original ones.

\subsection{Behavioral Performance Results}

The subject SAM-rating scores are listed in Table 5. When the results were examined, a significant difference was observed between the bright and original images in the arousal dimension, $(\mathrm{F}(1,30)=7.03, \mathrm{p}=0.013)$. However, no significant difference was observed in the valence dimension $(\mathrm{F}(1,30)=0.51, \mathrm{p}=0.48)$. According to the $t$-test results, the bright unpleasant images were considered as less arousing than the original images, $(p=0.001)$. However, there was no significant effect of brightness on the pleasant and neutral images. 
Table 5. Results of statistical evaluation of the valance and arousal score comparison between the original and bright version of pleasant, unpleasant and neutral images.

\begin{tabular}{|c|c|c|c|c|}
\hline $\begin{array}{l}\text { Visual } \\
\text { dimension }\end{array}$ & $\begin{array}{l}\text { Emotional } \\
\text { Conditions }\end{array}$ & $\begin{array}{l}\text { Original status } \\
(\text { mean } \pm s d)\end{array}$ & $\begin{array}{l}\text { Bright status } \\
(\text { mean } \pm \text { sd) }\end{array}$ & p-value \\
\hline \multirow{3}{*}{ valance } & Pleasant & $7.1 \pm 1.7$ & $7.1 \pm 1.7$ & 0.48 \\
\hline & Unpleasant & $3.4 \pm 1.4$ & $3.3 \pm 1.2$ & 0.50 \\
\hline & Neutral & $5.1 \pm 1.4$ & $5.1 \pm 1.3$ & 0.85 \\
\hline \multirow{3}{*}{ arousal } & Pleasant & $5.7 \pm 1.6$ & $5.8 \pm 1.6$ & 0.09 \\
\hline & Unpleasant & $5.7 \pm 1.6$ & $5.1 \pm 1.4$ & $0.001^{*}$ \\
\hline & Neutral & $3.6 \pm 1.6$ & $3.5 \pm 1.6$ & 0.67 \\
\hline
\end{tabular}

\section{Discussion}

In this study, the effects of image brightness were investigated on brain responses during visual processing of images with different emotional content. Time, frequency and location information, where brightness effect was observed, were examined using spectral power distributions of brain activities observed in EEG. In this study, it was focused on the differences between the average EEG power spectrograms obtained upon the presentation of the original and bright IAPS images. The main finding was that the brightness of visual stimuli affected neural activity. The average power of brain responses obtained upon the presentation of visual stimuli varied with the brightness of visual stimuli. This effect differed according to the time window, frequency band and electrode point where the activity was observed. Moreover, the brightness of visual stimuli on neural activity varied depending on the emotional content of the stimulus (pleasant, unpleasant and neutral). The findings revealed the frequency bands, time windows and electrode points, in which the brightness effect of visual stimuli was observed on brain activity for three different emotional contents (Figs. 5-7).

According to the results of the study, the response of the brain to the brightness of visual stimuli for the pleasant and unpleasant stimuli occurred with early time windows (100-200 ms) and continued until the late time windows $(700 \mathrm{~ms})$. However, for neutral visual stimuli, the brain's response to the brightness of these stimuli occurred later (200-300 ms) than other stimulus types and continued until the late time windows $(700 \mathrm{~ms})$. This means that brightness effect of visual stimulus appeared earlier than neutral images for pleasant and unpleasant images. The most noteworthy point among the findings was that at certain frequency bands and time windows, the increase in the brightness of the pleasant and neutral stimuli increased the average power of responses in the parietal and occipital regions, whereas the increase in brightness of the unpleasant stimuli decreased the average power of responses in the parietal and occipital regions. This effect was observed at high frequencies (alpha, beta and gamma) for the unpleasant and pleasant stimuli but at both low and 
distinctive effect of brightness occurred for all the three types of emotional stimuli in the parietal and occipital regions. The increase in the brightness of visual stimuli had a similar increasing effect on the power of the parietal and occipital responses for the pleasant and neutral images, but an opposite decreasing effect on the power of the unpleasant images. We know that the occipital lobe is the center of visual processing [51], containing neural communities that form the basis for visual perception. Information from the eye is processed in this lobe and associated with the information stored in memory [51]. Moreover, the parietal lobe processes sensory information for cognitive purposes. Another task of the parietal lobe is visual perception and recognition [51]. In other words, in our study, the distinctive effect of brightness for different types of stimuli appeared in visual fields. The increase in the brightness of visual stimuli may have a facilitating effect on the visual processing of neutral and pleasant images, while having a suppressive effect on the visual processing of unpleasant images.

However, for all the three types of stimuli, the increase in brightness reduced the average power of the frontal and central responses (except for 100-300 ms time window for unpleasant stimuli). This means that brightness caused the same effect on the anterior regions of the brain for all the three stimulus types. Unlike other stimuli for the unpleasant images, the increase in brightness increased the power of frontal beta responses in the 100-300 ms time window.

Previous studies have reported that frontal eye fields in the frontal cortex are important in directing attention toward targets in the visual field [52]. It is also known that frontal eye field responses have a sensory contribution to visual selection [53,54]. By taking this information into consideration, it can be suggested that the bright unpleasant stimuli compared with the original unpleasant stimuli may contribute to attention orientation by increasing the power of cortical activity in the early stages of visual processing. Of course, this effect should be investigated with further analysis.

Another noteworthy finding was that no effect of brightness was observed on the beta band for the neutral stimuli. Beta responses increase by high arousal, multisensory stimulation and cognitive load. Additionally, it has been reported that beta band activity reflects the arousal of the visual system with the increase of visual attention $[55,56]$. In the light of this information, it appears significant that there was no effect of brightness on brain activity for neutral images, which were less arousing than unpleasant and pleasant images.

Furthermore, the behavioral performance results appear to be consistent with our findings for the unpleasant images. According to the performance results, the bright unpleasant images were considered less arousing than their original counterparts. In many studies, it has been reported that arousal modulates brain activities mostly in late time periods (after 250ms) [21, 22, 57]. When Figs. (5-7) were examined, it was observed that the bright unpleasant images compared to their original counterparts decreased the power of brain activity in late time windows, as consistent with the performance results. This suppressive effect on brain activity may be due to arousal. Galvanic skin response measurements $[58,59]$ are required to demonstrate this effect clearly.

Our starting point in this study was the pioneering research, in which Lakens used IAPS images. Lakens reported that bright images were evaluated more positively and dark images were evaluated more negatively [35]. However, this evaluation was performed with behavioral data and how these findings are reflected in neural activity has not been so far investigated. Therefore, our primary objective in this study was to examine the brightness effect of images on the power of brain responses and determine the time, frequency and location characteristics of this effect. The findings revealed that the brightness of the image changed the power of brain responses to these images and that this effect differed according to the content of the emotional images. There have been many studies in the literature using IAPS and similar image. These studies are mostly in the fields of visual processing, emotion classification, emotion evaluation and neuro-marketing [4, 5, 9, 40,60-63]. Most of these studies did not consider the brightness of visual stimuli. However, our study results demonstrated that the brightness of visual stimuli was an important parameter with the potential to alter the power of brain activity. For this reason, researchers are required to take into account the brightness of visual 
stimuli in studies using visual stimuli. By controlling brightness effect, the misinterpretation of visual processing processes will be prevented. When the findings obtained in the literature are combined with the results obtained in the study, it is expected that the cognitive processes expected to be seen in the time windows where brightness effect occurs will also be affected by the brightness. This raises the question whether brightness can have an effect on the power of our emotions.

Visual perception is the result of physical properties affecting the visual system [64]. Since brightness, which is a physical feature of visual stimuli, affects this system, it can also create a different perception in the system. Although we have elucidated neurophysiological changes in the brain cortex due to brightness in this study, the cognitive interpretation of these changes still remain unclear.

In the early stages of visual information processing, brain oscillations respond to very basic features related to stimuli, while more complex and cognitive responses occur in the late stages. In many studies, time windows and frequency bands where these responses are observed have been reported $[6,8,12,13,25,65-67]$. However, in most of these studies, the brightness of visual stimuli was not considered. In other words, it is not clear whether sensory and cognitive processes are affected by the brightness of visual stimuli. Can the change in the power of neural activity by the brightness effect of visual stimuli affect the sensory and cognitive states (attention orientation, decision-making, memory) or the emotion we feel during these processes? In order to answer this question, advanced experimental mechanisms and advanced ERP analyses are needed. In future studies, by focusing on the time windows and frequency bands we specified in this study, important information can be obtained about brightness effect on emotional evaluation through advanced experimental mechanisms and ERP analyses. The detailed analysis of brightness effect on cognitive activity (directed attention, perception, memory, arousal, emotional evaluation, etc.) revealed that cognitive performance can be improved when brightness conditions are improved. This result will contribute positively to studies such as emotion classification and emotion analysis. In addition, the brightness of the image is an important issue for marketing science, which examines consumer behavior. Because emotions end with behavior and emotions that affect human behavior can be affected by brightness. Therefore, investigating the brightness effect of visual stimuli on cognitive performance will also contribute to neuro-marketing studies.

\section{Conclusions}

In this study, we demonstrated how brightness, a perceptual feature of pleasant, unpleasant and neutral emotional visual stimuli, affected neural activity during visual processing and how this effect was represented in the cortex. One of the original aspects of this study was that brightness effect on brain activity for visual stimuli with different emotional content was investigated in detail for different frequency bands and different channels at different time windows of visual processing. As shown in the findings, the brightness of visual stimuli has the potential to affect sensory and cognitive processes in visual processing studies. Therefore, we emphasize that brightness effect should be controlled in such studies. In our next study, we plan to investigate the effects of brightness on emotion categorization using time, frequency and location information, in which brightness is represented in the cortex.

Abbreviations: The following abbreviations are used in this manuscript;

IAPS: International affective picture system;

ERP: Event related potential;

EEG: Electroencephalography;

STFT: Short time Fourier transform;

C: Contrast value;

m: Meter;

EOG: Electrooculogram. 
Author Contributions: All authors contributed to the work presented in this paper. K.E.; data analysis, coding, writing, T.K.; methodology and O.O.; methodology. All authors read and approved the final manuscript.

Ethics approval and consent to participate: All subjects gave their informed consent for inclusion before they participated in the study. This study was conducted after the approval of the Ethics Committee of Istanbul Arel University by decision no 10432314-200.00.00-18.

Funding: This research received no external funding.

Acknowledgments: The authors would like to thank Pinar Kurt and the Psychophysiology Laboratory at Istanbul Arel University for their valuable help in the design of the experiments and collecting the EEG data.

Conflicts of Interest: The authors declare no conflict of interest.

\section{References}

Abhang, P. A., Gawali, B. W., \& Mehrotra, S. C. (2016). Introduction to Emotion, Electroencephalography, and Speech Processing. Introduction to EEG- and Speech-Based Emotion Recognition, 1-17. https://doi.org/10.1016/b978-0-12-804490-2.00001-4

Aftanas, L. I., Varlamov, A. A., Pavlov, S. V., Makhnev, V. P., \& Reva, N. V. (2002). Time-dependent cortical asymmetries induced by emotional arousal: EEG analysis of event-related synchronization and desynchronization in individually defined frequency bands. International Journal of Psychophysiology, 44(1), 67-82. https://doi.org/10.1016/S0167-8760(01)00194-5

Balconi, M., Brambilla, E., \& Falbo, L. (2009). Appetitive vs. defensive responses to emotional cues. Autonomic measures and brain oscillation modulation. Brain Research. https://doi.org/10.1016/j.brainres.2009.08.056

Balconi, M., Falbo, L., \& Brambilla, E. (2009). BIS/BAS responses to emotional cues: Self report, autonomic measure and alpha band modulation. Personality and Individual Differences, 47(8), 858863. https://doi.org/10.1016/j.paid.2009.07.004

Bamidis, P. D., Klados, M. A., Frantzidis, C., Vivas, A. B., Papadelis, C., Lithari, C., \& Pappas, C. (2009). A framework combining delta event-related oscillations (EROs) and synchronisation effects (ERD/ERS) to study emotional processing. Computational and Mathematical Methods in MedicineComputational Intelligence and Neuroscience. https://doi.org/10.1155/2009/549419

Bekhtereva, V., Craddock, M., \& Müller, M. M. (2015). Attentional bias to affective faces and complex IAPS images in early visual cortex follows emotional cue extraction. NeuroImage, 112, 254-266. https://doi.org/10.1016/j.neuroimage.2015.03.052

Bieniek, M. M., Frei, L. S., \& Rousselet, G. A. (2013). Early ERPs to faces: Aging, luminance, and individual differences. Frontiers in Psychology. https://doi.org/10.3389/fpsyg.2013.00268

Blanke, O., Morand, S., Thut, G., Michel, C. M., Spinelli, L., Landis, T., \& Seeck, M. (1999). Visual activity in the human frontal eye field, 10(5), 925-930.

Bradley, M. M., \& Lang, P. J. (1994). Measuring emotion: The self-assessment manikin and the semantic differential. Journal of Behavior Therapy and Experimental Psychiatry. https://doi.org/10.1016/0005-7916(94)90063-9

Clayson, P. E., \& Larson, M. J. (2019). The impact of recent and concurrent affective context on cognitive control: An ERP study of performance monitoring. International Journal of Psychophysiology, 143(March), 44-56. https://doi.org/10.1016/j.ijpsycho.2019.06.007 
Costa, T., Cauda, F., Crini, M., Tatu, M. K., Celeghin, A., De Gelder, B., \& Tamietto, M. (2014). Temporal and spatial neural dynamics in the perception of basic emotions from complex scenes. Social Cognitive and Affective Neuroscience, 9(11), 1690-1703. https://doi.org/10.1093/scan/nst164

Cuthbert, B. N., Schupp, H. T., Bradley, M. M., Birbaumer, N., \& Lang, P. J. (2000). Brain potentials in affective picture processing: covariation with autonomic arousal and affective report. Biological Psychology, 52(2), 95-111. https://doi.org/10.1016/S0301-0511(99)00044-7

Delplanque, S., Silvert, L., Hot, P., Rigoulot, S., \& Sequeira, H. (2006). Arousal and valence effects on event-related P3a and P3b during emotional categorization. International Journal of Psychophysiology. https://doi.org/10.1016/j.ijpsycho.2005.06.006

Ero, K., Bayram, T., \& Güntekin, B. (2017). The modulation of delta responses in the interaction of brightness and emotion, 112, 1-8. https://doi.org/10.1016/j.ijpsycho.2016.11.013

Feng, C., Wang, L., Liu, C., Zhu, X., Dai, R., Mai, X., \& Luo, Y. J. (2012a). The time course of the influence of valence and arousal on the implicit processing of affective pictures. PLoS ONE, 7(1), 19. https://doi.org/10.1371/journal.pone.0029668

Feng, C., Wang, L., Liu, C., Zhu, X., Dai, R., Mai, X., \& Luo, Y. J. (2012b). The time course of the influence of valence and arousal on the implicit processing of affective pictures. PLoS ONE. https://doi.org/10.1371/journal.pone.0029668

Ganin, I. P., Kosichenko, E. A., \& Kaplan, A. Y. (2018). Properties of EEG Responses to Emotionally Significant Stimuli Using a P300 Wave-Based Brain-Computer Interface. Neuroscience and Behavioral Physiology, 48(9), 1093-1099. https://doi.org/10.1007/s11055-018-0672-7

Gianotti, L. R. R., Faber, P. L., Schuler, M., Pascual-Marqui, R. D., Kochi, K., \& Lehmann, D. (2008). First valence, then arousal: The temporal dynamics of brain electric activity evoked by emotional stimuli. Brain Topography. https://doi.org/10.1007/s10548-007-0041-2

Güntekin, B., \& Başar, E. (2010a). A new interpretation of P300 responses upon analysis of coherences. Cognitive Neurodynamics. https://doi.org/10.1007/s11571-010-9106-0

Güntekin, B., \& Başar, E. (2010b). Event-related beta oscillations are affected by emotional eliciting stimuli. Neuroscience Letters. https://doi.org/10.1016/j.neulet.2010.08.002

Güntekin, B., \& Başar, E. (2014). A review of brain oscillations in perception of faces and emotional pictures. Neuropsychologia. https://doi.org/10.1016/j.neuropsychologia.2014.03.014

Güntekin, B., Emek-Savaş, D. D., Kurt, P., Yener, G. G., \& Başar, E. (2013). Beta oscillatory responses in healthy subjects and subjects with mild cognitive impairment. NeuroImage: Clinical. https://doi.org/10.1016/j.nicl.2013.07.003

Güntekin, B., Femir, B., Gölbaşı, B. T., Tülay, E., \& Başar, E. (2017). Affective pictures processing is reflected by an increased long-distance EEG connectivity. Cognitive Neurodynamics, 11(4), 355367. https://doi.org/10.1007/s11571-017-9439-z

Hess, R. F., \& Plant, G. T. (1983). The effect of temporal frequency variation on threshold contrast sensitivity deficits in optic neuritis. J Neurol.Neurosurg.Psychiatry, 46(4), 322-330.

Hot, P., \& Sequeira, H. (2013). Time course of brain activation elicited by basic emotions. NeuroReport, 24(16), 898-902. https://doi.org/10.1097/WNR.0000000000000016 
Jiang, X.-F., \& Bian, X.-P. (2013). Positive-Negative Emotional Categorization of Clothing Color Affect Hedonic Purchases? An ERPs Study. Frontiers in Neuroscience, 13(May), 1-9.

321 https://doi.org/10.3389/fnins.2019.00526 effects on early visual processing. Cognitive Brain Research. https://doi.org/10.1016/09266410(95)90008-X

Keil, A., Stolarova, M., Moratti, S., \& Ray, W. J. (2007). Adaptation in human visual cortex as a mechanism for rapid discrimination of aversive stimuli. NeuroImage, 36(2), 472-479. https://doi.org/10.1016/j.neuroimage.2007.02.048

Kim, C., Sun, J., Liu, D., Wang, Q., \& Paek, S. (2018). An effective feature extraction method by power spectral density of EEG signal for 2-class motor imagery-based BCI. Medical and Biological Engineering and Computing, 56(9), 1645-1658. https://doi.org/10.1007/s11517-017-1761-4

331 Lakens, D., Fockenberg, D. A., Lemmens, K. P. H., Ham, J., \& Midden, C. J. H. (2013). Brightness differences influence the evaluation of affective pictures. Cognition and Emotion, 27(7), 1225-1246. https://doi.org/10.1080/02699931.2013.781501

Lakens, D., Semin, G. R., \& Foroni, F. (2012). But for the bad, there would not be good: Grounding valence in brightness through shared relational structures. Journal of Experimental Psychology: General. https://doi.org/10.1037/a0026468 Technical manual and affective ratings. NIMH Center for the Study of Emotion and Attention, 3958.

340 Leyh, R., Heinisch, C., Kungl, M. T., \& Spangler, G. (2016). Attachment Representation Moderates 341 the Influence of Emotional Context on Information Processing. Frontiers in Human Neuroscience, 342 10(June), 1-12. https://doi.org/10.3389/fnhum.2016.00278

343 Li, W., \& Liu, Z. (2011). A method of SVM with normalization in intrusion detection. Procedia 344 Environmental Sciences, 11(PART A), 256-262. https://doi.org/10.1016/j.proenv.2011.12.040

345 Liu, N., Wang, K., Jin, X., Gao, B., Dellandréa, E., \& Chen, L. (2017). Visual affective classification by 346 combining visual and text features. PLoS ONE, 12(8), 6-9.

347 https://doi.org/10.1371/journal.pone.0183018

348 Liu, W., Liu, F., Chen, L., Jiang, Z., \& Shang, J. (2019). Cognitive reappraisal in children: 349 Neuropsychological evidence of up-regulating positive emotion from an ERP study. Frontiers in 350 Psychology, 10(FEB), 1-10. https://doi.org/10.3389/fpsyg.2019.00147 (2012). The dynamics of EEG gamma responses to unpleasant visual stimuli: From local activity to functional connectivity. NeuroImage, 60(2), 922-932.

355 https://doi.org/10.1016/j.neuroimage.2012.01.060

356 Mavratzakis, A., Herbert, C., \& Walla, P. (2016). Emotional facial expressions evoke faster orienting 
358

359

360

361

362

363

364

365

366

367

368

369

370

371

372

373

374

375

376

377

378

379

380

381

382

383

384

385

386

387

388

389

390

391

392

simultaneous EEG and facial EMG study. NeuroImage.

https://doi.org/10.1016/j.neuroimage.2015.09.065

McFarland, D. J., Parvaz, M. A., Sarnacki, W. A., Goldstein, R. Z., \& Wolpaw, J. R. (2017). Prediction of subjective ratings of emotional pictures by EEG features. Journal of Neural Engineering, 14(1), 19. https://doi.org/10.1088/1741-2552/14/1/016009

McManus, C. (1995). Cognitive Assessment for Clinicians. BMJ, 76. https://doi.org/10.1136/bmj.310.6979.608

Meier, B. P., Robinson, M. D., Crawford, L. E., \& Ahlvers, W. J. (2007). When "light" and "dark" thoughts become light and dark responses: Affect biases brightness judgments. Emotion. https://doi.org/10.1037/1528-3542.7.2.366

Migliore, S., Curcio, G., Porcaro, C., Cottone, C., Simonelli, I., D’aurizio, G., ... Vernieri, F. (2019). Emotional processing in RRMS patients: Dissociation between behavioural and neurophysiological response. Multiple Sclerosis and Related Disorders, 27(October 2018), 344-349.

https://doi.org/10.1016/j.msard.2018.11.019

Miskovic, V., \& Schmidt, L. A. (2010). Cross-regional cortical synchronization during affective image viewing. Brain Research, 1362, 102-111. https://doi.org/10.1016/j.brainres.2010.09.102

Modica, E., Cartocci, G., Perrotta, D., Di Feo, P., Aricò, P., Inguscio, B. M. S., ... Mancini, M. (2018).

Neurophysiological profile of antismoking campaigns. Computational Intelligence and

Neuroscience, 2018. https://doi.org/10.1155/2018/9721561

MONTAGU, J. D., \& COLES, E. M. (1968). Mechanism and Measurement of the Galvanic Skin Response: an Addendum. Psychological Bulletin, 69(1), 74-76. https://doi.org/10.1037/h0025305

Muggleton, N. G. (2003). Human Frontal Eye Fields and Visual Search. Journal of Neurophysiology. https://doi.org/10.1152/jn.01086.2002

Müller, M. M., \& Gundlach, C. (2017). Competition for attentional resources between low spatial frequency content of emotional images and a foreground task in early visual cortex.

Psychophysiology, 54(3), 429-443. https://doi.org/10.1111/psyp.12792

Olofsson, J. K., Nordin, S., Sequeira, H., \& Polich, J. (2008). Affective picture processing: An integrative review of ERP findings. Biological Psychology.

https://doi.org/10.1016/j.biopsycho.2007.11.006

Olofsson, J. K., \& Polich, J. (2007). Affective visual event-related potentials: Arousal, repetition, and time-on-task. Biological Psychology. https://doi.org/10.1016/j.biopsycho.2006.12.006

Peli, E. (1990). Contrast in complex images. Journal of the Optical Society of America, 7(10), 20322040. https://doi.org/10.1364/JOSAA.7.002032

Rozenkrants, B., \& Polich, J. (2008). Affective ERP processing in a visual oddball task: Arousal, valence, and gender. Clinical Neurophysiology. https://doi.org/10.1016/j.clinph.2008.07.213

Sánchez-Reolid, R., García, A., Vicente-Querol, M., Fernández-Aguilar, L., López, M., \& González, A. (2018). Artificial Neural Networks to Assess Emotional States from Brain-Computer Interface. Electronics, 7(12), 384. https://doi.org/10.3390/electronics7120384 
Sanei, S., \& Chambers, J. A. (2007). EEG Signal Processing. In EEG/ERP Analysis (pp. 35-126). England: John Wiley \& Sons,Ltd. https://doi.org/10.1002/9781118622162.ch2

Schettino, A., Keil, A., Porcu, E., \& Müller, M. M. (2016). Shedding light on emotional perception: Interaction of brightness and semantic content in extrastriate visual cortex. NeuroImage, 133, 341353. https://doi.org/10.1016/j.neuroimage.2016.03.020

Singh, M. I., \& Singh, M. (2017). Development of a real time emotion classifier based on evoked EEG. Biocybernetics and Biomedical Engineering, 37(3), 498-509. https://doi.org/10.1016/j.bbe.2017.05.004

Smith, N. K., Cacioppo, J. T., Larsen, J. T., \& Chartrand, T. L. (2003). May I have your attention, please: Electrocortical responses to positive and negative stimuli. Neuropsychologia (Vol. 41).

Sobolewski, A., Holt, E., Kublik, E., \& Wróbel, A. (2011). Impact of meditation on emotional processing-A visual ERP study. Neuroscience Research. https://doi.org/10.1016/j.neures.2011.06.002

Valberg, A. (2005). Light Vision Color. Light Vision Color. John Wiley \& Sons.

Valdez, P., \& Mehrabian, A. (1994). Effects of Color on Emotions. Journal of Experimental Psychology: General, 123(4), 394-409.

Wróbel, A. (2000). Beta activity: a carrier for visual attention. Acta Neurobiol. Exp, 60(2), 247-260.

Yu Shi, A., Ruiz, N., Taib, R., C Choi, E. H., \& Chen, F. (2007). Galvanic Skin Response (GSR) as an Index of Cognitive Load CHI 2007 @BULLET Work-in-Progress. Chi, 2651-2656.

Yuan, J., Zhang, Q., Chen, A., Li, H., Wang, Q., Zhuang, Z., \& Jia, S. (2007). Are we sensitive to valence differences in emotionally negative stimuli? Electrophysiological evidence from an ERP study. Neuropsychologia. https://doi.org/10.1016/j.neuropsychologia.2007.04.018

Yusoff, N., Anuar, N. N., \& Reza, M. F. (2018). The effect of sex on the electropsychological process of emotional arousal intensity. Malaysian Journal of Medical Sciences, 25(3), 103-110.

https://doi.org/10.21315/mjms2018.25.3.10

Zamuner, E. (2013). The Role of the Visual System in Emotion Perception. Acta Analytica, 28(2), 179-187. https://doi.org/10.1007/s12136-012-0151-7

Zhang, Q., \& Lee, M. (2012). Emotion development system by interacting with human EEG and natural scene understanding. Cognitive Systems Research.

https://doi.org/10.1016/j.cogsys.2010.12.012

Zhang, W., Lu, J., Liu, X., Fang, H., Li, H., Wang, D., \& Shen, J. (2013). Event-related synchronization of delta and beta oscillations reflects developmental changes in the processing of affective pictures during adolescence. International Journal of Psychophysiology, 90(3), 334-340. https://doi.org/10.1016/j.ijpsycho.2013.10.005. 